\title{
Semiclassical Analysis of Discretizations of Schrödinger-type Equations
}

\author{
PETER A. MARKOWICH ${ }^{\mathrm{a}, *}$, PAOLA PIETRA $^{\mathrm{b}, \dagger}$ and CARSTEN POHL PA $^{\mathrm{a},}$ \\ ${ }^{a}$ Johannes Kepler Universität Linz, Institut für Analysis und Numerik, Abtl. Differentialgleichungen, Altenberger Str. 69, A-4040 Linz, \\ Austria; ${ }^{\mathrm{b}}$ Istituto di Analisi Numerica del C. N. R., Via Abbiategrasso 209, I-27100 Pavia, Italy
}

(Received 13 August 1997; In final form 1 December 1998)

\begin{abstract}
We apply Wigner-transform techniques to the analysis of difference methods for Schrödinger-type equations in the case of a small Planck constant. In this way we are able to obtain sharp conditions on the spatial-temporal grid which guarantee convergence for average values of observables as the Planck constant tends to zero. The theory developed in this paper is not based on local and global error estimates and does not depend on whether caustics develop or not.

Numerical examples are presented to help interpret the theory.
\end{abstract}

Keywords: Discretization of Schrödinger equation, Wigner transform

\section{INTRODUCTION}

Many problems of solid-state physics require the (numerical) solution of the Schrödinger equation in the case of a small (scaled) Planck constant $\varepsilon$ :

$$
\begin{gathered}
\varepsilon u_{t}^{\varepsilon}-i \frac{\varepsilon^{2}}{2} \Delta u^{\varepsilon}+i V(x) u^{\varepsilon}=0, \quad x \in \mathbb{R}^{m}, \quad t \in \mathbb{R} \\
u^{\varepsilon}(x, t=0)=u_{I}^{\varepsilon}(x), \quad x \in \mathbb{R}^{m} .
\end{gathered}
$$

Here $V$ is a given electrostatic potential, $0<\varepsilon \ll 1$ and $u^{\varepsilon}=u^{\varepsilon}(x, t)$ is the wave function. By classical quantum physics [10] the wave function is an auxiliary quantity used to compute the primary physical quantities, which are quadratic function(al)s of $u^{\varepsilon}(t), e . g$. , the position density

$$
n^{\varepsilon}(x, t)=\left|u^{\varepsilon}(x, t)\right|^{2}
$$

the current density

$$
\left.J^{\varepsilon}(x, t)=\varepsilon \operatorname{Im} \overline{\left(u^{\varepsilon}(x, t)\right.} \nabla u^{\varepsilon}(x, t)\right),
$$

(where "-" denotes complex conjugation). It is well known that the Eq. (1.1a) propagates oscillations of wave length $\varepsilon$, which inhibit $u^{\varepsilon}$ from converging strongly in, say, $L_{t}^{\infty}\left(L_{x}^{2}\right)$. Clearly, the weak

*Tel.: +43 (0)732-2468 9190, e-mail: markowic@math.tu-berlin.de

${ }^{\dagger}$ Tel.: +390382 529600, e-mail: pietra@dragon.ian.pv.cnr.it

${ }^{\ddagger}$ Corresponding author. Tel.: +43 (0) 732-2468 9187, e-mail: carsten.pohl@jk.uni-linz.ac.at 
convergence of $u^{\varepsilon}$ is, for example, not sufficient for passing to the limit in the macroscopic densities (1.2), which implies that the analysis of the socalled semi-classical limit is a mathematically rather complex issue.

Recently, much progress has been made in this area, particularly by the introduction of tools from microlocal analysis (defect measures [5], H-measures [19] and Wigner measures [6, 11, 13, 7]). These techniques, which go far beyond classical WKBmethods, have shown the right way to exploit properties of the Schrödinger equation which, despite the oscillatory nature, allow the passage to the limit $\varepsilon \rightarrow 0$ in the macroscopic densities by revealing an underlying kinetic structure.

Exactly the same problem, i.e., the highly oscillatory nature of the solutions, has to be coped with when the Schrödinger equation with small $\varepsilon$ is solved numerically. Even for stable discretization schemes (or under mesh size restrictions which guarantee stability) the oscillations may very well pollute the solution in such a way that the quadratic macroscopic quantities (1.2) and other physical observables come out completely wrong when the scaled Planck constant is small.

For the linear Schrödinger equation classical numerical analysis methods (like the stabilityconsistency concept) are sufficient to derive meshing strategies for discretizations which guarantee (locally) strong convergence of the discrete wave functions to $u^{\varepsilon}$ when $\varepsilon>0$ is fixed (cf. [21,1-3]). Extensions to nonlinear Schrödinger equations can be found in $[4,20,16,18]$.

However, the classical strategies cannot be employed to analyze properties of discretization schemes for $\varepsilon \rightarrow 0$.

In this paper we adapt the microlocal techniques used to analyze the semi-classical limit for the IVP (1.1) to the analysis of finite difference discretizations. We choose a sample of time discretizations: the Crank-Nicolson scheme and the Leap-Frog scheme (both belonging to the set of mostly used methods for the Schrödinger equation). Corresponding spatial discretizations are general arbitrary-order symmetric schemes. For these methods we identify the semiclassical Wigner measure (on the scale $\varepsilon$ ) for all (sensible) combinations of $\varepsilon$ and of the time and space mesh sizes. We clearly have convergence for the average values of all (regular) observables in those cases, for which the Wigner measure of the numerical scheme is identical to the Wigner measure of the Schrödinger equation itself. Thus, from this theory we obtain sharp (i.e., necessary and sufficient) conditions on the mesh sizes which guarantee good approximation quality of all (smooth) observables for $\varepsilon$ small. We remark that the approach is sharp because we do not use local and global error estimates.

The theory and the presented test calculations clearly show the big risk in doing Schrödinger calculations for small Planck constant. Even highly stable schemes might produce completely wrong observables under seemingly reasonable meshing strategies (i.e., asymptotic resolution of the oscillation is not always enough). Worse enough, in these cases there is no warning from the scheme (like blow-up) that something went wrong (since local error control cannot be used anyway). It seems that the only safety anchor here lies in the analysis and, for more difficult problems than the linear Schrödinger equation, in physical insight.

The paper is organized as follows. In Section 2 we present the basic analytical tools (e.g., Wigner transforms) needed to carry out the program on the discretization schemes, Section 3 is concerned with formulating the finite difference schemes in the language of pseudo-differential calculus and Section 4 contains the identification of the Wigner measures of the discretizations. Numerical sample computations and interpretations of the theory are given in Section 5.

\section{SCHRÖDINGER-TYPE EQUATIONS, OBSERVABLES AND WIGNER TRANSFORMS}

We consider the following scalar IVP

$$
\varepsilon u_{t}^{\varepsilon}+i Q(x, \varepsilon D)^{W} u^{\varepsilon}=0, \quad x \in \mathbb{R}^{m}, \quad t \in \mathbb{R}
$$




$$
u^{\varepsilon}(x, t=0)=u_{I}^{\varepsilon}(x), \quad x \in \mathbb{R}^{m} .
$$

Here $\varepsilon \in\left(0, \varepsilon_{0}\right], \varepsilon_{0}>0$, is a small parameter (e.g., the scaled Planck-constant), $Q(\cdot, \varepsilon D)^{W}$ is the Weyloperator associated to the symbol $Q(x, \varepsilon \xi)$ :

$$
\begin{aligned}
& Q(x, \varepsilon D)^{W} \varphi(x) \\
& \quad:=\frac{1}{(2 \pi)^{m}} \int_{\mathbb{R}_{y}^{m}} \int_{\mathbb{R}_{\xi}^{m}} Q\left(\frac{x+y}{2}, \varepsilon \xi\right) \varphi(y) \mathrm{e}^{i(x-y) \cdot \xi} d \xi d y .
\end{aligned}
$$

For the following we assume that the symbol $Q=Q(x, \xi)$ is polynomial in $\xi$ with $C^{\infty}$-coefficients:

$$
Q(x, \xi)=\sum_{|k| \leq K} Q_{k}(x) \xi^{k},
$$

where $k=\left(k_{1}, \ldots, k_{m}\right) \in \mathbb{N}_{0}^{m}$ denotes a multiindex, $K$ is the order of the differential operator (2.2) and $|k|:=k_{1}+\cdots+k_{m}$ the order of the multiindex $k$. The DO (2.2) can now be written as

$$
\begin{aligned}
& Q(x, \varepsilon D)^{W} \varphi(x) \\
& \quad=\left.\sum_{|k| \leq K} \varepsilon^{|k|} D_{y}^{k}\left(Q_{k}\left(\frac{x+y}{2}\right) \varphi(y)\right)\right|_{y=x} .
\end{aligned}
$$

We denoted $D_{y}=-i \partial_{y}$.

The convenience in the Weyl-calculus lies in the fact that a Weyl-operator is formally selfadjoint iff it has a real valued symbol ( $c f$. [8]).

Since we are interested in generalizations of the Schrödinger-equation we assume for the following

(i) $Q_{k}$ is real valued for $0 \leq|k| \leq K$,

(ii) $\forall k, \alpha \in \mathbb{N}_{0}^{m}$ with $|k| \leq K \exists C_{k, \alpha}>0$ :

$$
\left|\partial_{x}^{\alpha} Q_{k}(x)\right| \leq C_{k, \alpha} \forall x \in \mathbb{R}^{m} \text {. }
$$

This implies in particular that $Q(x, \varepsilon D)^{W}$ is selfadjoint on $L^{2}\left(\mathbb{R}^{m}\right)$.

By Stone's Theorem $\exp \left(-i(t / \varepsilon) Q(\cdot, \varepsilon D)^{W}\right)$ is a strongly continuous group of unitary operators on $L^{2}\left(\mathbb{R}^{m}\right)$. Thus we conclude the $L^{1}\left(\mathbb{R}^{m}\right)$-conservation in time of the so-called position-density $n^{\varepsilon}(x, t):=\left|u^{\varepsilon}(x, t)\right|^{2}$ :

$$
\int_{\mathbb{R}^{m}} n^{\varepsilon}(x, t) d x=\int_{\mathbb{R}^{m}} n_{I}^{\varepsilon}(x) d x \quad \forall t \in \mathbb{R}
$$

where we set $n_{I}^{\varepsilon}:=\left|u_{I}^{\varepsilon}\right|^{2}$.
In quantum mechanics the wave function $u^{\varepsilon}=u^{\varepsilon}(x, t)$ (i.e., the solution of the Schrödingerequation) is usually considered an auxiliary quantity. It facilitates the calculation of physical observables of the system under consideration [10] corresponding to actual measurements. An observable $A^{\varepsilon}$, which depends on the position variable $x$ and on the momentum operator $\varepsilon D$, is given by the Weyl-operator

$$
A^{\varepsilon}=a(\cdot, \varepsilon D)^{W}
$$

with the real valued symbol $a(x, \varepsilon \xi)$. Of particular physical interest is the average value of the observable $A^{\varepsilon}$ in the state $u^{\varepsilon}(t)$ (i.e., the mean value of the performed measurement) given by:

$$
E_{a}^{\varepsilon}(t):=\left(a(\cdot, \varepsilon D)^{W} u^{\varepsilon}(t), u^{\varepsilon}(t)\right) .
$$

Here $(\cdot, \cdot)$ stands for the $L^{2}\left(\mathbb{R}^{m}\right)$-scalar product and, of course, it is assumed that $u^{\varepsilon}(t)$ lies in the domain of $a(\cdot, \varepsilon D)^{W}$.

A good framework for manipulating quantities which are quadratic in the wave function (e.g. (2.7)), is given by the Wigner-transform [7, 22]. For given functions $f, g \in \mathcal{S}^{\prime}\left(\mathbb{R}^{m}\right)$ and a given scale $\varepsilon \in\left(0, \varepsilon_{0}\right]$ we define the Wigner-transform (on the scale $\varepsilon$ ) by

$$
\begin{aligned}
& w^{\varepsilon}(f, g)(x, \xi) \\
& \quad=\frac{1}{(2 \pi)^{m}} \int_{\mathbb{R}^{m}} f\left(x-\varepsilon \frac{v}{2}\right) \overline{g\left(x+\varepsilon \frac{v}{2}\right)} \mathrm{e}^{i v \cdot \xi} d v .
\end{aligned}
$$

For fixed $\varepsilon$ this defines a bilinear continuous mapping from $\mathcal{S}^{\prime}\left(\mathbb{R}^{m}\right) \times \mathcal{S}^{\prime}\left(\mathbb{R}^{m}\right)$ into $\mathcal{S}^{\prime}\left(\mathbb{R}_{x}^{m} \times \mathbb{R}_{\xi}^{m}\right)$. By a simple calculation we have

$$
\left\langle w^{\varepsilon}(f, g), a\right\rangle=\left\langle\bar{g}, a(\cdot, \varepsilon D)^{W} f\right\rangle .
$$

Here we assume $a \in \mathcal{S}\left(\mathbb{R}_{x}^{m} \times \mathbb{R}_{\xi}^{m}\right)$ and denote by $\langle\cdot, \cdot\rangle$ the duality bracket between $\mathcal{S}^{\prime}$ and $\mathcal{S}$ (linear in both arguments). Obviously, (2.9) implies

$$
E_{a}^{\varepsilon}(t)=\int_{\mathbb{R}_{x}^{m} \times \mathbb{R}_{\xi}^{m}} w^{\varepsilon}\left(u^{\varepsilon}(t), u^{\varepsilon}(t)\right)(x, \xi) a(x, \xi) d x d \xi
$$


The proof of the following theorem can be found in [15].

Theorem 2.1 Let $Q \in C^{\infty}\left(\mathbb{R}_{x}^{m} \times \mathbb{R}_{\xi}^{m}\right)$ satisfy for some $M \geq 0, C_{\alpha} \geq 0$ :

$$
\begin{aligned}
\partial_{x, \xi}^{\alpha} Q(x, \xi) \mid \leq & C_{\alpha}(1+|\xi|)^{M} \\
& \forall \alpha \in \mathbb{N}_{0}^{m} \times \mathbb{N}_{0}^{m}
\end{aligned}
$$

and assume that the initial datum $u_{I}^{\varepsilon}$ of (2.1) satisfies

$$
u_{I}^{\varepsilon} \in L^{2}\left(\mathbb{R}^{m}\right) \text { uniformly as } \varepsilon \rightarrow 0 .
$$

Then there exists $w^{0} \in L^{\infty}\left(\mathbb{R}_{t} ; \mathcal{M}^{+}\left(\mathbb{R}_{x}^{m} \times \mathbb{R}_{\xi}^{m}\right)\right)$ (where $\mathcal{M}^{+}$stands for the cone of positive Borelmeasures) such that after selection of a subsequence

$$
w^{\varepsilon} \stackrel{\varepsilon \rightarrow 0}{\longrightarrow} w^{0} \quad \text { in } L^{\infty}\left(\mathbb{R}_{t} ; \mathcal{M}^{+}\left(\mathbb{R}_{x}^{m} \times \mathbb{R}_{\xi}^{m}\right)\right) \text { weak-* }
$$

and $w^{0}$ satisfies the transport equation

$$
\frac{\partial}{\partial t} w^{0}+\left\{Q, w^{0}\right\}=0, \quad(x, \xi) \in \mathbb{R}_{x}^{m} \times \mathbb{R}_{\xi}^{m}, \quad t \in \mathbb{R}
$$

subject to the initial condition

$$
w^{0}(t=0)=w_{I}^{0}:=w^{0}\left(u_{I}^{\varepsilon}, u_{I}^{\varepsilon}\right) .
$$

In (2.13a) $\{\cdot, \cdot\}$ denotes the Poisson bracket:

$$
\{f, g\}=\partial_{\xi} f \cdot \partial_{x} g-\partial_{x} f \cdot \partial_{\xi} g .
$$

Note that the convergence (2.12) holds for the whole sequence $w^{\varepsilon}$ if $w_{I}^{0}$ is the unique Wignermeasure of $u_{I}^{\varepsilon}$ (i.e., independent of the subscale $\varepsilon_{k}$ ).

The unique solution of (2.13) then allows the calculation of the limit $\varepsilon \rightarrow 0$ of the average value of an observable $A^{\varepsilon}$ determined by a symbol $a=a(x, \xi) \in \mathcal{S}$ in the state $u^{\varepsilon}(c f$. (2.7) and (2.10)). We obtain

$$
E_{a}^{\varepsilon} \rightarrow E_{a}^{0}:=\int a(x, \xi) w^{0}(d x, d \xi, t)
$$

after selection of a subsequence. Since the limit process (2.12) is actually locally uniform in $t$ [7], the convergence (2.14) takes place in $C_{\text {loc }}\left(\mathbb{R}_{t}\right)$.

Easy calculations give for the position density

$$
n^{\varepsilon}(x, t)=\int_{\mathbb{R}_{\xi}^{m}} w^{\varepsilon}(x, \xi, t) d \xi .
$$

Additional assumptions on the initial datum $u_{I}^{\varepsilon}$ have to be imposed in order to be able to pass to the limit $\varepsilon \rightarrow 0$ in (2.15) (note that (2.14) cannot be applied directly since $n^{\varepsilon}$ corresponds to a momentum independent observable, whose symbol is not in $S\left(\mathbb{R}_{x}^{m} \times \mathbb{R}_{\xi}^{m}\right)$ ). A complete account of this is given in [7]. Here we only remark that

$$
n^{\varepsilon} \longrightarrow n^{0}:=\int_{\mathbb{R}_{\xi}^{m}} w^{0}(x, d \xi, t)
$$

holds if $u_{I}^{\varepsilon}$ is $\varepsilon$-oscillatory, i.e.,

$$
\varlimsup_{\varepsilon \rightarrow 0} \int_{|\xi| \geq \frac{R}{\varepsilon}}\left|\widehat{\varphi u_{I}^{\varepsilon}}(\xi)\right|^{2} d \xi \stackrel{R \rightarrow \infty}{\longrightarrow} 0
$$

for every continuous, compactly supported function $\varphi$. The assumption (2.17) says that $u_{I}^{\varepsilon}$ oscillates with wavelength at least $O(\varepsilon)$.

In order to avoid taking subsequences we shall assume for the following

$$
w_{I}^{0} \text { is the unique Wigner-measure of } u_{I}^{\varepsilon} \text {. }
$$

\section{FINITE DIFFERENCE SCHEMES}

Let $\Gamma=\left\{\mu=l_{1} a_{1}+\cdots+l_{m} a_{m} \mid l_{j} \in \mathbb{Z}\right.$ for $\left.1 \leq j \leq m\right\}$ $\subseteq \mathbb{R}^{m}$ be the lattice generated by the linearly independent vectors $a_{1}, \ldots, a_{m} \in \mathbb{R}^{m}$. For a multiindex $k \in \mathbb{N}_{0}^{m}$ we construct a discretization of the order $N$ of the operator $\partial_{x}^{k}$ as follows:

$$
\partial_{x}^{k} \varphi(x) \sim \frac{1}{h^{|k|}} \sum_{\mu \in \Gamma_{k}} a_{\mu, k} \varphi(x+h \mu) .
$$

Here $h \in\left(0, h_{0}\right]$ is the mesh-size, $\Gamma_{k} \subseteq \Gamma$ is the finite set of discretization points and $a_{\mu, k} \in \mathbb{R}$ are 
coefficients satisfying

$$
\sum_{\mu \in \Gamma_{k}} a_{\mu, k} \mu^{l}=\delta_{l, k} k !, \quad 0 \leq|l| \leq N+|k|-1
$$

where $\delta_{l, k}=1$ if $l=k$ and 0 otherwise. It is an easy exercise to show that the local discretization error of (3.1) is $O\left(h^{N}\right)$ for all smooth functions if (D1) holds. For a detailed discussion of the linear problem (D1) (i.e., possible choices of the coefficients $a_{\mu, k}$ ) we refer to [14].

Given the discretization (3.1) for $0 \leq|k| \leq K$ we now define the corresponding finite difference discretization of $Q(\cdot, \varepsilon D)^{W}$ by applying (3.1) (with $\partial=i D)$ directly to (2.4).

Denoting

$$
\begin{aligned}
Q_{h, \varepsilon}(x, \xi) & =\sum_{|k| \leq K} \varrho^{|k|}(-i)^{|k|} \sum_{\mu \in \Gamma_{k}} a_{\mu, k} e^{i \frac{\mu}{e} \cdot \xi} Q_{k}(x), \\
\varrho & =\frac{\varepsilon}{h}
\end{aligned}
$$

we obtain the finite difference discretization of (2.4) in the form

$$
\begin{aligned}
& Q_{h, \varepsilon}(x, \varepsilon D)^{W} \varphi(x) \\
& \quad=\sum_{|k| \leq K} \varrho^{|k|}(-i)^{|k|} \sum_{\mu \in \Gamma_{k}} a_{\mu, k} Q_{k}\left(x+\frac{h \mu}{2}\right) \varphi(x+h \mu) .
\end{aligned}
$$

Since $Q_{h, \varepsilon}(x, \varepsilon D)^{W}$ is a bounded operator on $L^{2}\left(\mathbb{R}^{m}\right)$, it is selfadjoint if

$i^{|k|} \sum_{\mu \in \Gamma_{k}} a_{\mu, k} \mathrm{e}^{i \mu \cdot \xi}$ is real valued for $0 \leq|k| \leq K$.

As temporal discretizations we consider the onestep schemes with time step $\Delta t>0$ :

$$
\begin{gathered}
\varepsilon \frac{u_{n+1}^{\sigma}-u_{n}^{\sigma}}{\Delta t}+i Q_{h, \varepsilon}(x, \varepsilon D)^{W}\left(\alpha u_{n+1}^{\sigma}+\beta u_{n}^{\sigma}\right) \\
=0, \quad n=0,1,2, \ldots \\
u_{0}^{\sigma}=u_{I}^{\varepsilon}
\end{gathered}
$$

with $\alpha \geq 0, \beta \geq 0, \alpha+\beta=1$. Here (and in the sequel) we denote the vector of small parameters by $\sigma=(\varepsilon, h, \Delta t) . u_{n}^{\sigma}$ is the obtained approximation of $u^{\varepsilon}\left(t_{n}\right)$ where $t_{n}=n \Delta t, n \in \mathbb{N}_{0}$. In this paper we will restrict ourselves to the analysis of the CrankNicolson scheme $(\alpha=\beta=(1 / 2))$. The (non timereversible and non mass-conserving) implicit/ explicit Euler schemes are not investigated here but the interested reader may find the analysis in [15].

Also we shall analyze the Leap-Frog method

$$
\begin{aligned}
\varepsilon \frac{u_{n+2}^{\sigma}-u_{n}^{\sigma}}{2 \Delta t}+i Q_{h, \varepsilon}(x, \varepsilon D)^{W} u_{n+1}^{\sigma} \\
=0, \quad n=0,1,2, \ldots \\
u_{0}^{\sigma}=u_{I}^{\varepsilon}, \quad u_{1}^{\sigma}=\tilde{u}_{1}^{\sigma}
\end{aligned}
$$

The choice of $\tilde{u}_{1}^{\sigma}$ will be discussed later on.

Note that the selfadjointness of $Q_{h, \varepsilon}(x, \varepsilon D)^{W}$ implies that the operator $I d+i \omega Q_{h, \varepsilon}(x, \varepsilon D)^{W}$ is boundedly invertible on $L^{2}\left(\mathbb{R}_{x}^{m}\right)$ for all $\omega \in \mathbb{R}$. Therefore also the scheme (3.4) for $\alpha>0$ gives welldefined approximations $u_{n}^{\sigma}$ for $n=1,2, \ldots$ if $u_{I}^{\varepsilon} \in L^{2}\left(\mathbb{R}_{x}^{m}\right)$. Moreover we remark that it is sufficient to evaluate (3.4) and, resp., (3.5) at $x \in h \Gamma$ in order to obtain discrete equations for $\left\{u_{n}^{\sigma}(h \mu) \mid\right.$ $\mu \in \Gamma\}$. Clearly, artificial 'far out' boundary conditions have to be imposed for practical computations. Their impact will not be taken into account in the subsequent analysis.

We now collect properties of the finite difference schemes. We start with the spatial discretization:

LEMMA 3.1 Let (A1), (D1), (D2) hold and assume that $\varphi \in \mathcal{S}\left(\mathbb{R}_{x}^{m} \times \mathbb{R}_{\xi}^{m}\right)$. For $\varrho=\frac{\varepsilon}{h} \stackrel{\varepsilon, h \rightarrow 0}{\longrightarrow} \infty$ we have

$$
Q_{h, \varepsilon} \varphi \stackrel{\varepsilon, h \rightarrow 0}{\longrightarrow} Q \varphi \quad \text { in } \mathcal{S}\left(\mathbb{R}_{x}^{m} \times \mathbb{R}_{\xi}^{m}\right) .
$$

\section{Proof see [15].}

Choosing $h$ such that $\varrho=(\varepsilon / h) \rightarrow \infty$ corresponds to asymptotically resolving the oscillations of wave-length $O(h)$ of the solution $u^{\varepsilon}(t)$ of (2.1). In the case $\varrho=$ const. (i.e., 'placing a fixed number of gridpoints per oscillation') the symbol $Q_{h, \varepsilon}(x, \xi)$ is independent of $h$ and $\varepsilon$ :

$$
\begin{aligned}
Q_{h, \varepsilon}(x, \xi) & \equiv Q_{\varrho}(x, \xi) \\
& :=\sum_{|k| \leq K} \varrho^{|k|} \sum_{\mu \in \Gamma_{k}} a_{\mu, k}(-i)^{|k|} \mathrm{e}^{i(\mu / \varrho) \cdot \xi} Q_{k}(x) .
\end{aligned}
$$


In the case $\varrho^{\varepsilon, h \rightarrow 0 /} \longrightarrow 0$ (which corresponds to not resolving the oscillations asymptotically) we have

$$
Q_{h, \varepsilon} \stackrel{h, \varepsilon \rightarrow 0}{\sim} \sum_{\mu \in \Gamma_{0}} a_{\mu, 0} \cos \left(\frac{\mu \cdot \xi}{\varrho}\right) Q_{0}(x)
$$

and, thus, $Q_{h, \varepsilon}(x, \varepsilon D)^{W}$ does not approximate $Q(x, \varepsilon D)^{W}$. Therefore, we cannot expect reasonable numerical results in this case (which will not be investigated further).

The Crank-Nicolson scheme is unconditionally stable. The Leap-Frog scheme is stable if

$$
\Delta t<d \frac{\varepsilon h^{K}}{\varepsilon^{K}+h^{K}}
$$

for some $d>0$ sufficiently small.

\section{CONVERGENCE OF WIGNER MEASURES}

The consistency-stability concept of classical numerical analysis provides a framework for the convergence analysis of finite difference discretizations of linear partial differential equations. Thus, for $\varepsilon>0$ fixed it is easy to prove that the schemes (3.4) and, resp., (3.5) (with an appropriate choice of $\tilde{u}_{1}^{\sigma}$ ) are convergent of order $N$ in space and order 2 in time if the solution $u^{\varepsilon}$ is sufficiently smooth and if the stability constraints on the meshsizes $\Delta t$ and $h$ of Lemma 3.1 are taken into account. Therefore, again for fixed $\varepsilon>0$ we conclude convergence of the same order for averages of the observables defined in (2.7) assuming that $a$ is smooth. Due to the oscillatory nature of the solutions of (2.1) the local discretization error of the finite difference schemes and, consequently, also the global discretization error, generally tend to infinity as $\varepsilon$ tends to 0 . The situation is still complicated by the fact that - again due to the occurrence of $O(\varepsilon)$-wavelength oscillations - the solution $u^{\varepsilon}$ of (2.1) and their discrete approximations $u_{n}^{\sigma}$, which solve (3.4) or, resp., (3.5), generally only converge weakly in $L^{2}\left(\mathbb{R}^{m}\right)$ as $\varepsilon \rightarrow 0$ and, resp., $\sigma \rightarrow 0$. The limit processes $\varepsilon \rightarrow 0, \sigma \rightarrow 0$ do not commute with the quadratically nonlinear operation which has to be carried out to compute the average values of observables. In practice one is interested in finding (mild) conditions on the mesh sizes $h$ and $\Delta t$, in dependence of $\varepsilon$ and the used discretization such that the average values of the observables in the discrete state converge (for $\varepsilon$ small) to $E_{a}^{\varepsilon}$ given by (2.7).

Let us set for $n \in \mathbb{N}, t_{n}=n \Delta t$ :

$$
E_{a}^{\sigma}\left(t_{n}\right):=\left(a(\cdot, \varepsilon D)^{W} u_{n}^{\sigma}, u_{n}^{\sigma}\right) .
$$

The function $E_{a}^{\sigma}(t), t \in \mathbb{R}^{+}$, then is defined by piecewise linear interpolation of the values $E_{a}^{\sigma}\left(t_{n}\right)$.

We want to find conditions on $h, \Delta t$ (in the form, say, $\left.\sigma \in B \subseteq \mathbb{R}^{3}\right)$ such that for all $a \in \mathcal{S}\left(\mathbb{R}_{x}^{m} \times \mathbb{R}_{\xi}^{m}\right)$ :

$$
\lim _{\substack{\sigma \rightarrow 0 \\ \sigma \in B}} E_{a}^{\sigma}(t)=E_{a}^{0}(t)
$$

locally uniformly in $t$. Denoting

$$
w^{\sigma}\left(t_{n}\right):=w^{\varepsilon}\left(u_{n}^{\sigma}, u_{n}^{\sigma}\right)
$$

and again defining $w^{\sigma}(t), t \in \mathbb{R}$, by piecewise linear interpolation of the values (4.2), we conclude from (2.10) that (4.1b) is equivalent to proving

$$
\lim _{\substack{\sigma \rightarrow 0 \\ \sigma \in B}} w^{\sigma}(t)=w^{0}(t) \quad \text { in } \mathcal{S}^{\prime}\left(\mathbb{R}_{x}^{m} \times \mathbb{R}_{\xi}^{m}\right)
$$

locally uniformly in $t$, where $w^{\varepsilon}=w^{\varepsilon}\left(u^{\varepsilon}, u^{\varepsilon}\right)$ is the Wigner-transform of the solution $u^{\varepsilon}$ of (2.1). Note that $w^{\sigma}\left(t_{n}\right)$ denotes the Wigner-transform of the finite difference solution $u_{n}^{\sigma}$ on the scale $\varepsilon$.

In this Section we shall compute the accumulation points of $w^{\sigma}$ as $\sigma \rightarrow 0$. We shall see that the set of Wigner-measures of the difference schemes

$$
\begin{array}{r}
A:=\left\{W^{0} \mid \exists \text { subsequence }\left(\sigma_{l}\right) \text { of }(\sigma)\right. \\
\left.: W^{0}=\lim _{l \rightarrow \infty} w^{\sigma_{l}}\right\}
\end{array}
$$

depends decisively on the discretization method and on the relative sizes of $\varepsilon, h$ and $\Delta t$. In those cases, in which $W^{0}=w^{0}\left(=\lim _{\varepsilon \rightarrow 0} w^{\varepsilon}\right)$ holds, (4.3) follows for $\sigma_{l} \in B$, while (4.3) does not hold if the measures $W^{0}$ and $w^{0}$ are different. 
We start with the Crank-Nicolson scheme

TheOREM 4.1 Let $\alpha=\beta=(1 / 2)$ and assume that (A1), (A2), (A3), (D1), (D2) hold. Then the following cases occur for the unique Wigner-measure $W^{0} \in A$ of (3.4):

Case $1 \quad h / \varepsilon \rightarrow 0(\varrho \rightarrow \infty)$.

(i) $(\Delta t / \varepsilon) \rightarrow 0$. Then $W^{0}$ satisfies:

$$
\frac{\partial}{\partial t} W^{0}+\left\{Q, W^{0}\right\}=0, W^{0}(t=0)=w_{I}^{0}
$$

(ii) $(\Delta t / \varepsilon) \rightarrow \omega \in \mathbb{R}^{+} . W^{0}$ solves the IVP:

$$
\begin{aligned}
\frac{\partial}{\partial t} W^{0}+\left\{\frac{2}{\omega} \arctan \left(\frac{\omega}{2} Q\right), W^{0}\right\} & =0, \\
W^{0}(t=0) & =w_{I}^{0}
\end{aligned}
$$

(iii) $(\Delta t / \varepsilon) \rightarrow \infty$. If there exists $D>0$ such that:

$$
|Q(x, \xi)| \geq D \quad \forall x, \xi \in \mathbb{R}^{m}
$$

then $W^{0}$ is constant in time

$$
W^{0}(x, \xi, t) \equiv W_{I}^{0}(x, \xi)
$$

Case $2 \quad(h / \varepsilon) \rightarrow(1 / \varrho) \in \mathbb{R}^{+}$. Then the assertions (i), (ii), (iii) hold true, if $Q$ is replaced by $Q_{\varrho}$ in (4.5), (4.6), (4.7a).

We recall that $Q_{\varrho}$ is defined in (3.7).

Corollary 4.1 In addition to the assumptions of Theorem 4.1 assume that $u_{I}^{\varepsilon}$ is $\varepsilon$-oscillatory. Then we have

$$
n^{\sigma}:=\int_{\mathbb{R}_{\xi}^{m}} w^{\sigma} d \xi \stackrel{\sigma \rightarrow 0}{\longrightarrow} N^{0}:=\int_{\mathbb{R}_{\xi}^{m}} W^{0}(x, d \xi, t)
$$

Now we state the result for the Leap-Frog scheme:

Theorem 4.2 Let (A1), (A2), (A3), (D1), (D2) hold and let the stability condition (3.8) be satisfied. Also let $\tilde{u}_{1}^{\sigma}$ be uniformly bounded in $L^{2}\left(\mathbb{R}_{x}^{m}\right)$ and assume that $\lim _{\sigma \rightarrow 0} w^{\varepsilon}\left(\tilde{u}_{1}^{\sigma}, u_{I}^{\varepsilon}\right)$ exists in $\mathcal{S}^{\prime}\left(\mathbb{R}_{x}^{m} \times \mathbb{R}_{\xi}^{m}\right)$. Set $z_{I}^{0}:=\operatorname{Re} \lim _{\sigma \rightarrow 0} w^{\varepsilon}\left(\tilde{u}_{1}^{\sigma}, u_{I}^{\varepsilon}\right)$. Then the following cases occur for the solution of the Leap-Frog scheme (3.5):

Case $1 \quad(h / \varepsilon) \rightarrow 0 \quad(\varrho \rightarrow \infty)$. Then the unique Wigner-measure $W^{0} \in A$ satisfies:

$$
\frac{\partial}{\partial t} W^{0}+\left\{Q, Z^{0}\right\}=0, \quad W^{0}(t=0)=w_{I}^{0}
$$

$$
\begin{gathered}
\frac{\partial}{\partial t} Z^{0}+\left\{Q, W^{0}\right\}=0, \quad Z^{0}(t=0)=z_{I}^{0} \\
W^{0}(t=0)=w_{I}^{0}, \quad Z^{0}(t=0)=z_{I}^{0}
\end{gathered}
$$

Case $2 \quad(h / \varepsilon) \rightarrow(1 / \varrho) \in \mathbb{R}^{+}$.

(i) $(\Delta t / \varepsilon) \rightarrow 0$. Then the assertion of (1) remains true if $Q$ is replaced by $Q_{\varrho}$ in (4.9).

(ii) $(\Delta t / \varepsilon) \rightarrow \omega \in \mathbb{R}^{+}$. Then every Wigner-measure $W^{0} \in A$ satisfies:

$$
\begin{aligned}
\left(1-\omega^{2} Q_{\varrho}^{2}\right) \frac{\partial}{\partial t} W^{0}+\left\{Q_{\varrho}, Z^{0}\right\} & =0, \\
W^{0}(t=0) & =w_{I}^{0}
\end{aligned}
$$

$$
\begin{gathered}
\frac{\partial}{\partial t} Z^{0}+\left\{Q_{\varrho}, W^{0}\right\}=0, \quad Z^{0}(t=0)=z_{I}^{0} \\
W^{0}(t=0)=w_{I}^{0}, \quad Z^{0}(t=0)=z_{I}^{0}
\end{gathered}
$$

If $\tilde{u}_{1}^{\sigma}$ is chosen such that $z_{I}^{0}=w_{I}^{0}$ (e.g., setting $\tilde{u}_{1}^{\sigma}=u_{I}^{\varepsilon}$ ) then (4.9) gives $Z^{0} \equiv W^{0}$ and the transport equation

$$
\frac{\partial}{\partial t} W^{0}+\left\{Q, W^{0}\right\}=0, \quad W^{0}(t=0)=w_{I}^{0}
$$

follows in Theorem 4.2, Case (1). Again, for (2) (i) we have to replace $Q$ by $Q_{\varrho}$.

We remark that the convergence of $w^{\sigma}$ to $W^{0}$ is locally uniform in $t$ in Theorem 4.1, Theorem 4.2, Cases (1) and (2) (i) while it is only in $L^{\infty}\left(\mathbb{R}_{t}, \mathcal{S}^{\prime}\right)$ weak-* in the Case (2) (ii) of Theorem 4.2. In the latter case we cannot generally exclude the 
occurrence of more than one accumulation point of $w^{\sigma}$.

The two Theorems give necessary and sufficient conditions for

$$
A=\left\{w^{0}=w^{0}\left[u^{\varepsilon}\right]\right\}
$$

i.e., for the property of the difference schemes that their Wigner-measures are equal to the Wignermeasure associated to the IVP (2.1). Summarizing, these conditions are:

(1) Crank-Nicolson scheme: $(\Delta t / \varepsilon) \rightarrow 0,(h / \varepsilon) \rightarrow 0$.

(2) Leap-Frog scheme: $(h / \varepsilon) \rightarrow 0$ and $\Delta t \leq d\left(\left(\varepsilon h^{K}\right) /\right.$ $\left.\left(\varepsilon^{K}+h^{K}\right)\right)$ for some $d>0$ sufficiently small.

In all other cases there are initial data $u_{I}^{\varepsilon}$ for which either instabilities occur or such that the Wigner-measure of the difference scheme under consideration is different from the Wigner-measure of the Schrödinger-type IVP.

It can be shown that the term $1-\omega^{2} Q_{\varrho}^{2}$ (appearing in (4.10a)) is strictly positive if the stability condition (3.8) is satisfied.

The proofs of the Theorems can be found in [15].

\section{EXAMPLES AND NUMERICAL RESULTS}

We consider the linear Schrödinger equation (already in scaled form) which describes the transport of a charged particle under the influence of an electrostatic potential $V(x) \in C^{\infty}\left(\mathbb{R}^{m}\right)$ with uniformly bounded derivatives

$$
\begin{gathered}
\varepsilon u_{t}^{\varepsilon}-i \frac{\varepsilon^{2}}{2} \Delta u^{\varepsilon}+i V(x) u^{\varepsilon}=0, \quad x \in \mathbb{R}^{m}, t \in \mathbb{R} \\
u^{\varepsilon}(x, t=0)=u_{I}^{\varepsilon}(x), \quad x \in \mathbb{R}^{m} .
\end{gathered}
$$

Here $\varepsilon$ is the scaled Planck constant. In this case the symbol $Q$ is simply

$$
Q(x, \xi)=\frac{1}{2}|\xi|^{2}+V(x) .
$$

The initial condition (5.1b) is chosen in WKB form

$$
u_{I}^{\varepsilon}(x)=\sqrt{n_{I}(x)} \exp \left(\frac{i}{\varepsilon} S_{I}(x)\right), \quad x \in \mathbb{R}^{m},
$$

with $n_{I}(x)$ and $S_{I}(x)$ independent of $\varepsilon$, real valued, regular and with $n_{I}(x)$ decaying to zero sufficiently fast as $|x| \rightarrow \infty$.

As already pointed out, the main goal when solving the Schrödinger equation is to compute macroscopic quantities associated to the wave function $u^{\varepsilon}$. The most important macroscopic quantities are the position density $n^{\varepsilon}$ and the current density $J^{\varepsilon}$ :

$$
\begin{aligned}
n^{\varepsilon}(x, t) & :=\left|u^{\varepsilon}(x, t)\right|^{2}, \\
J^{\varepsilon}(x, t) & :=\varepsilon \operatorname{Im}\left(\overline{u^{\varepsilon}(x, t)} \nabla u^{\varepsilon}(x, t)\right) .
\end{aligned}
$$

The (weak) limits as $\varepsilon \rightarrow 0$ of $n^{\varepsilon}$ and $J^{\varepsilon}$ can be completely characterized using the Wigner measure theory $([11,12,7])$. The Wigner measure of the wave function $u^{\varepsilon}(t)$ is the solution of the Liouville equation (see (2.13a) with $Q(x, \xi)$ given by (5.2)),

$$
\begin{gathered}
\frac{\partial}{\partial t} w^{0}(x, \xi, t)+\xi \cdot \nabla_{x} w^{0}(x, \xi, t) \\
-\nabla_{x} V(x) \cdot \nabla_{\xi} w^{0}(x, \xi, t)=0,
\end{gathered}
$$

subject to the initial condition (obtained from (5.3) by a simple computation)

$$
w_{I}^{0}(x, \xi)=n_{I}(x) \delta\left(\xi-\nabla S_{I}(x)\right) .
$$

Then, conservation of charge gives

$$
n^{\varepsilon} \longrightarrow n^{0}:=\int_{\mathbb{R}_{\xi}^{m}} w^{0}(x, d \xi, t),
$$

and conservation of energy implies

$$
J^{\varepsilon} \longrightarrow J^{0}:=\int_{\mathbb{R}_{\xi}^{m}} \xi w^{0}(x, d \xi, t) .
$$

Details on proving (5.5) can be found in [7].

The test problem (5.1) is considered in the one dimensional case and it is discretized in space with the usual three-point symmetric scheme, and in time with the Crank-Nicolson scheme given by (3.4) or with the Leap-Frog scheme given by (3.5). 
The finite difference operator is

$$
\begin{aligned}
& Q_{h, \varepsilon}(x, \varepsilon D)^{W} \varphi(x) \\
& \quad=-\frac{\varepsilon^{2}}{2} \frac{\varphi(x-h)-2 \varphi(x)+\varphi(x+h)}{h^{2}}+V(x) \varphi(x),
\end{aligned}
$$

with symbol

$$
Q_{h, \varepsilon}(x, \xi)=\frac{\varepsilon^{2}}{h^{2}}\left(1-\cos \frac{h \xi}{\varepsilon}\right)+V(x) .
$$

The space discretization is consistent of order 2 (i.e., (D1) is satisfied with $N=2$ ) and has a real valued symbol (thus (D2) is fulfilled). We recall that condition (D2) is a condition on the symmetry of the discretization. It implies that the Weyl operator is selfadjoint.

We denote by $u_{j, n}^{\sigma}$ the approximation of $u^{\varepsilon}$ $\left(x_{j}, t_{n}\right)$, where $t_{n}=n \Delta t, x_{j}=j h$ is a given mesh point and $\sigma=(\varepsilon, h, \Delta t)$. We recall the definition of the discrete position density and current density

$$
\begin{array}{r}
n^{\sigma}\left(x_{j}, t_{n}\right):=\left|u_{j, n}^{\sigma}\right|^{2}, n=0,1, \ldots, x_{j} \in \Gamma, \\
J^{\sigma}\left(x_{j}, t_{n}\right):=\varepsilon \operatorname{Im}\left(\frac{u_{j, n}^{\sigma}}{\left.\frac{u_{j+1, n}^{\sigma}-u_{j, n}^{\sigma}}{h}\right),}\right. \\
n=0,1, \ldots, x_{j} \in \Gamma .
\end{array}
$$

We want to investigate in which situations the computed quantities $n^{\sigma}$ and $J^{\sigma}$ converge (weakly) to $n^{0}$ and $J^{0}$, as $\sigma \rightarrow 0$. Therefore, in the pictures presented in the following $n^{\sigma}$ and $J^{\sigma}$ are plotted and compared with $n^{0}$ and $J^{0}$. As an example, Figures $4-6$ show a sequence of $n^{\sigma}$ and $J^{\sigma}$ converging to $n^{0}$ and $J^{0}$. Most of the other figures illustrate cases where the sequence does not converge to $n^{0}$ and $J^{0}$. The answer is ( partially) given by Theorems 4.1 and 4.2. They give conditions on the mesh sizes $h$ and $\Delta t$ to guarantee that the Wigner measure of the discrete scheme $W^{0}$ (defined in (4.4)) coincides with the Wigner measure of the continuous problem $w^{0}$ (defined in (5.4)). As a direct consequence, all the observables of the finite difference scheme under consideration converge to the exact observables.
Cases where $W^{0}$ does not converge to $w^{0}$, but $n^{\sigma}$ converges to $n^{0}$ are possible. In the continuous case, $n^{0}$ and $J^{0}$ are recovered as moments of the Wigner measure (see (5.5)). In the discrete case the situation is more delicate. For the Crank-Nicolson scheme, Corollary 4.1 states that $n^{\sigma} \stackrel{\sigma \rightarrow 0}{\longrightarrow} N^{0}:=\int_{\mathbb{R}_{\varepsilon}^{m}} W^{0}$ $(x, d \xi, t)$ if the initial datum $u_{I}^{\varepsilon}$ is $\varepsilon$-oscillatory. As already mentioned, the WKB initial datum (5.3) implies the $\varepsilon$-oscillatory property, so if $W^{0}$ coincides with $w^{0}$, then convergence of $n^{\sigma}$ to $n^{0}$ is guaranteed, otherwise $n^{\sigma}$ can converge to something else. The main ingredient to prove Corollary 4.1 is that the $\varepsilon$-oscillatory property of the initial datum is preserved by the scheme (and for Crank-Nicolson this follows from the $L^{2}$-conservation). For the Leap-Frog scheme the $\varepsilon$-oscillatory property is not preserved in general, because of the lack of $L^{2}$-conservation. However, in the case of constant coefficient operators the $\varepsilon$-oscillatory property of the solution is preserved in time. Thus, an analogous result as in Corollary 4.1 holds true in this case for all the time discretization schemes considered here. Moreover, for constant coefficient operators a complete characterization of the convergence of the current density is possible. (see [12])

In all the numerical tests presented here, the computations are carried out in the interval $[0,1]$ and periodic boundary conditions are used. The potential $V=0$ is chosen, unless otherwise specified.

In Figures $1-18$ the initial condition (5.3) is taken with

$$
\begin{aligned}
n_{I}(x) & =\left(\exp \left(25(x-0.5)^{2}\right)\right)^{2} \\
\frac{d}{d x} S_{I}(x) & =-\tanh (5(x-0.5))
\end{aligned}
$$

(see [9]). The initial condition is plotted in Figure 1. Due to the compressive initial velocity $v_{I}(x)=(d / d x) S_{I}(x), n^{0}$ and $J^{0}$ are $L_{\text {loc }}^{1}$ functions with singularities on the caustics that develop at time $t=0.2$ (for a more detailed describtion see [15]). The weak limits $n^{0}(x, t)$ and $J^{0}(x, t)$ are plotted in Figures 2 and 3 for $t=0.15$ (before the caustics develop) and $t=0.54$ (after the caustics develop). For $\varepsilon>0, n^{\varepsilon}$ and $J^{\varepsilon}$ oscillate with 

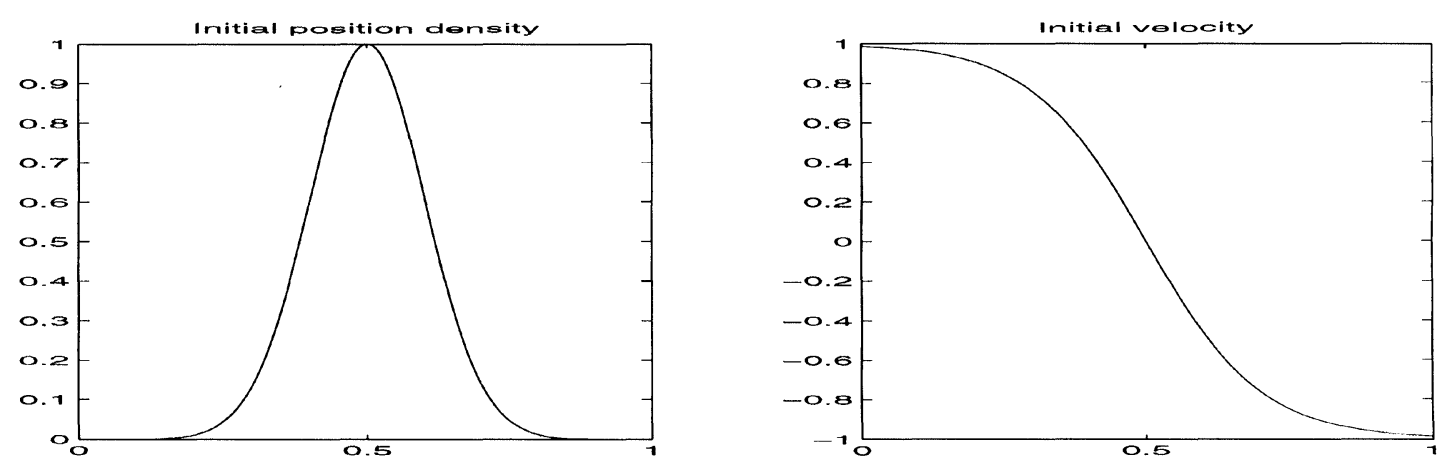

FIGURE 1 Initial condition, $n_{I}(x)$ and $u_{I}(x)=\partial_{x} S_{I}(x)$.
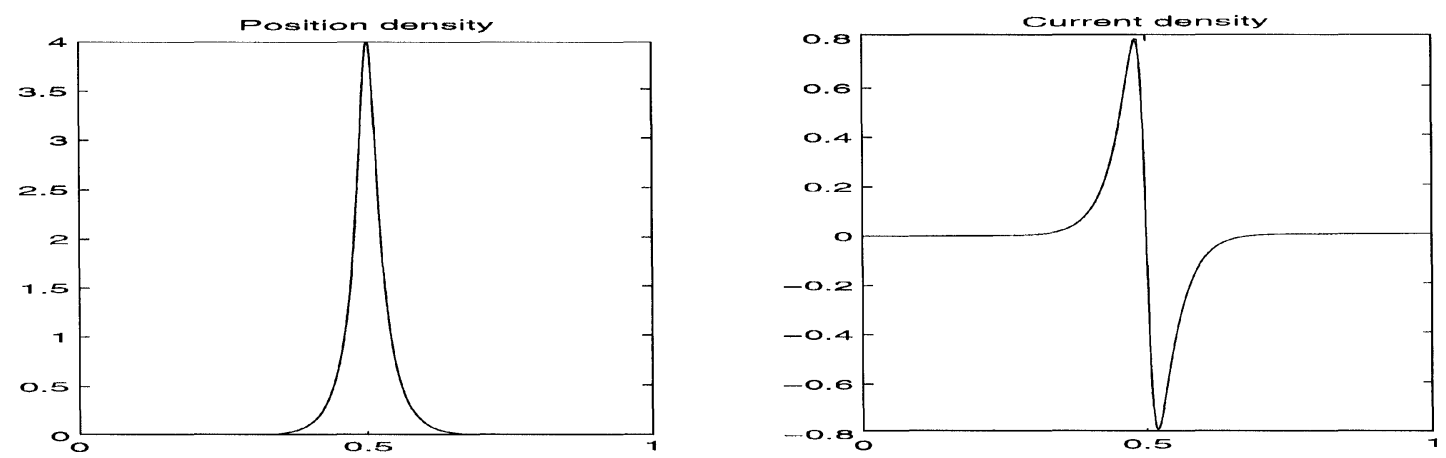

FIGURE 2 Weak limits $n^{0}(x, 0.15)$ and $J^{0}(x, 0.15)$.
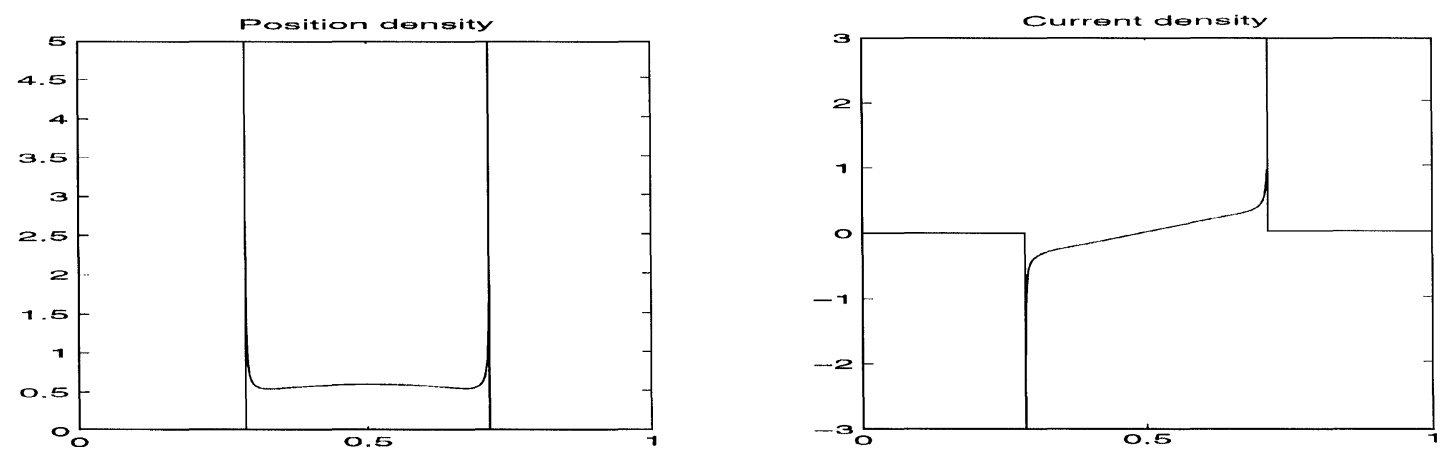

FIGURE 3 Weak limits $n^{0}(x, 0.54)$ and $J^{0}(x, 0.54)$.

wavelength $O(\varepsilon)$ (see [9]) in the region between the caustics.

All the pictures presented in the following show $n^{\sigma}$ and $J^{\sigma}$ at time $t=0.54$ (after caustics have developed). The reference $n^{0}$ and $J^{0}$ are the ones of Figure 3.
Crank-Nicolson The first set of tests refers to the Crank-Nicolson time discretization (3.4) with $\alpha=\beta=0.5$. The Crank-Nicolson scheme is one of the most widely used finite difference schemes for the discretization of the Schrödinger equation. The main reason lies in its conservation properties: 

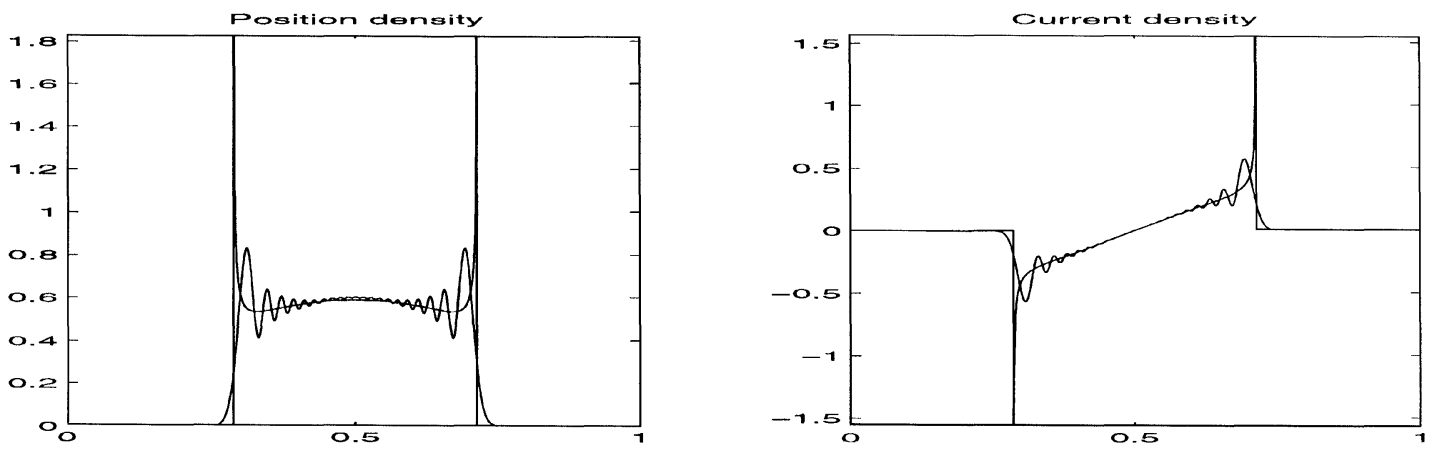

FIGURE 4 Crank-Nicolson, $\varepsilon=2 \cdot 10^{-3}, \Delta t=\varepsilon^{1.5}, h=\varepsilon^{1.2}, V=0$.
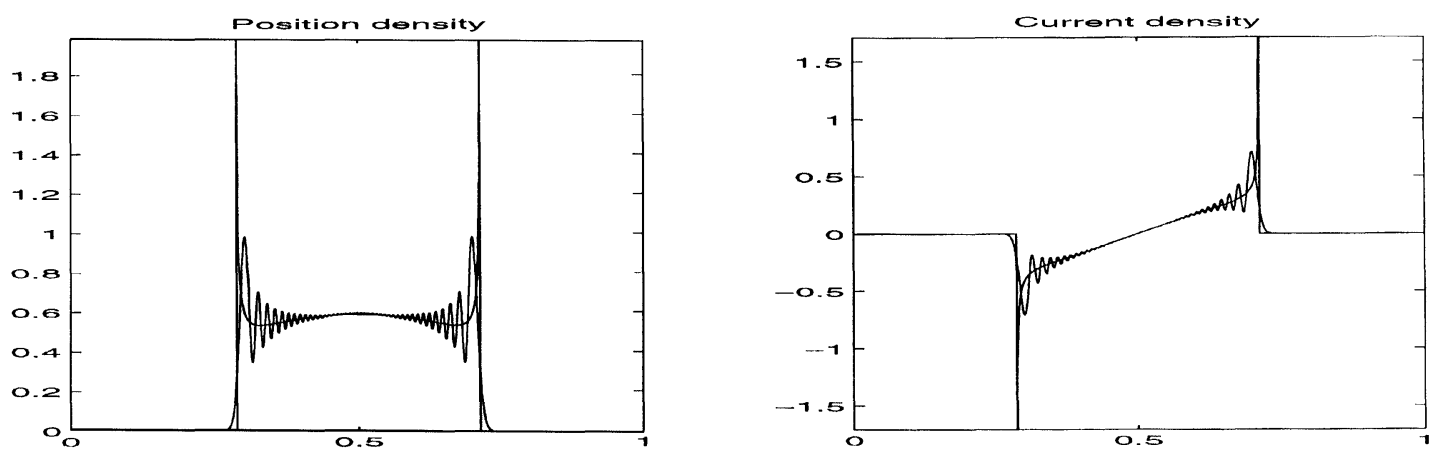

FIGURE 5 Crank-Nicolson, $\varepsilon=10^{-3}, \Delta t=\varepsilon^{1.5}, h=\varepsilon^{1.2}, V=0$.
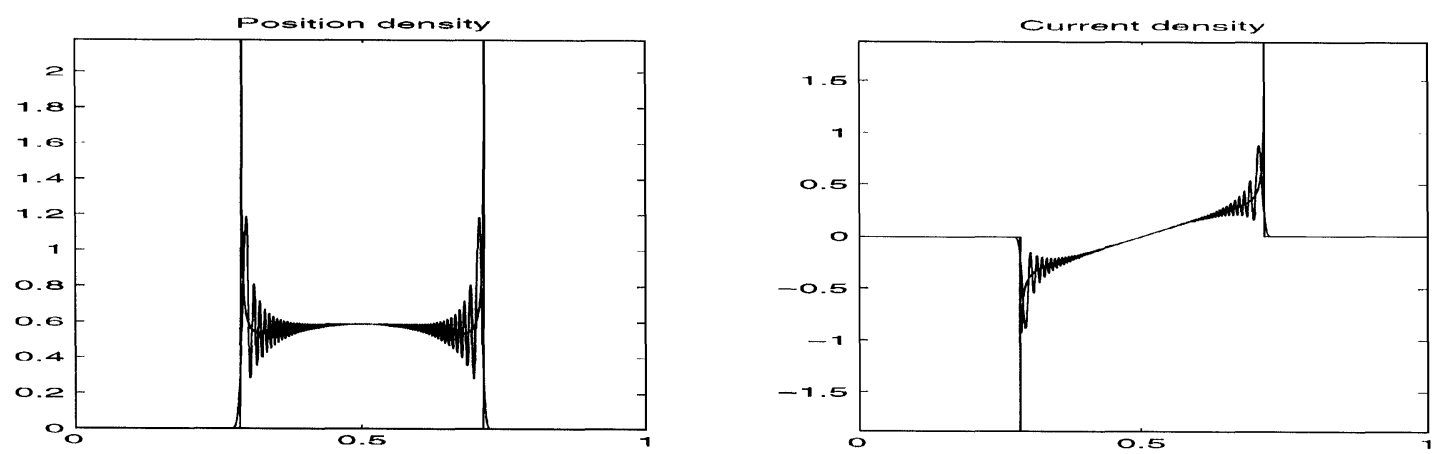

FIGURE 6 Crank-Nicolson, $\varepsilon=0.5 \cdot 10^{-3}, \Delta t=\varepsilon^{1.5}, h=\varepsilon^{1.2}, V=0$.

the position density and the total energy $\left(\left(\varepsilon^{2} / 2\right)\right.$ $\left.\int\left|\nabla_{x} u^{\sigma}\left(x, t^{n}\right)\right|^{2} d x+\int V(x)\left|u^{\sigma}\left(x, t^{n}\right)\right|^{2} d x\right)$ are preserved. These conservation properties are important, but by far not enough to guarantee good convergence for small $\varepsilon$.
Figures 4-6 show $n^{\sigma}$ and $n^{0}\left(J^{\sigma}\right.$ and $J^{0}$, resp.) with $h=\varepsilon^{1.2}, \Delta t=\varepsilon^{1.5}$ and $\varepsilon=2 \cdot 10^{-3}, \varepsilon=10^{-3}$, $\varepsilon=0.5 \cdot 10^{-3}$. The discretization parameters $h$ and $\Delta t$ satisfy the hypotheses (1) (i) of Theorem 4.1 , therefore the Wigner measure of the numerical 

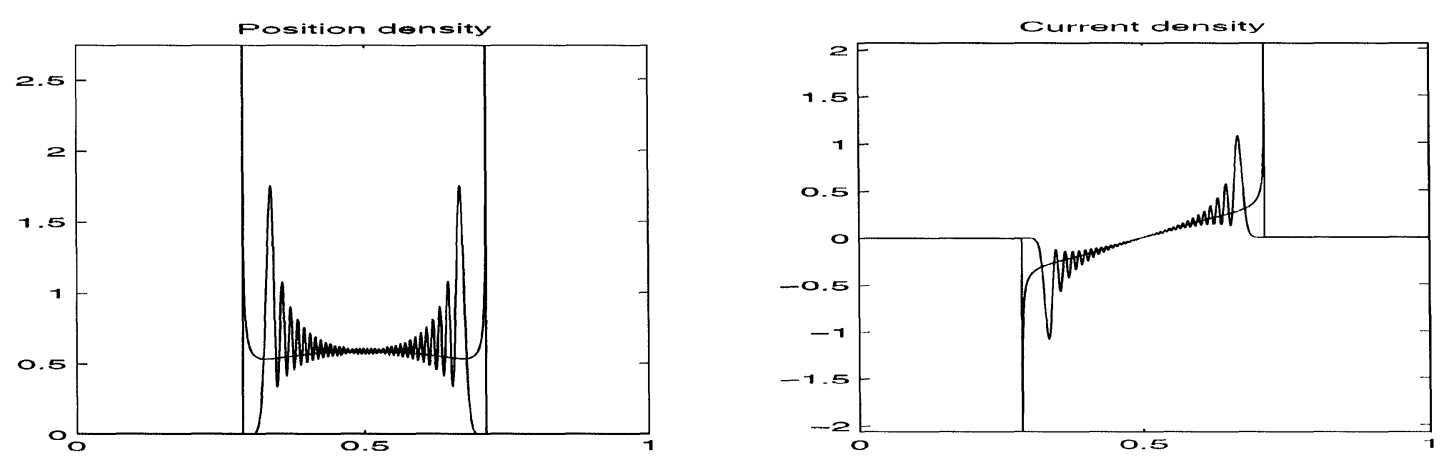

FIGURE 7 Crank-Nicolson, $\varepsilon=10^{-3}, \Delta t=\varepsilon^{1.5}, h=\varepsilon, V=0$.
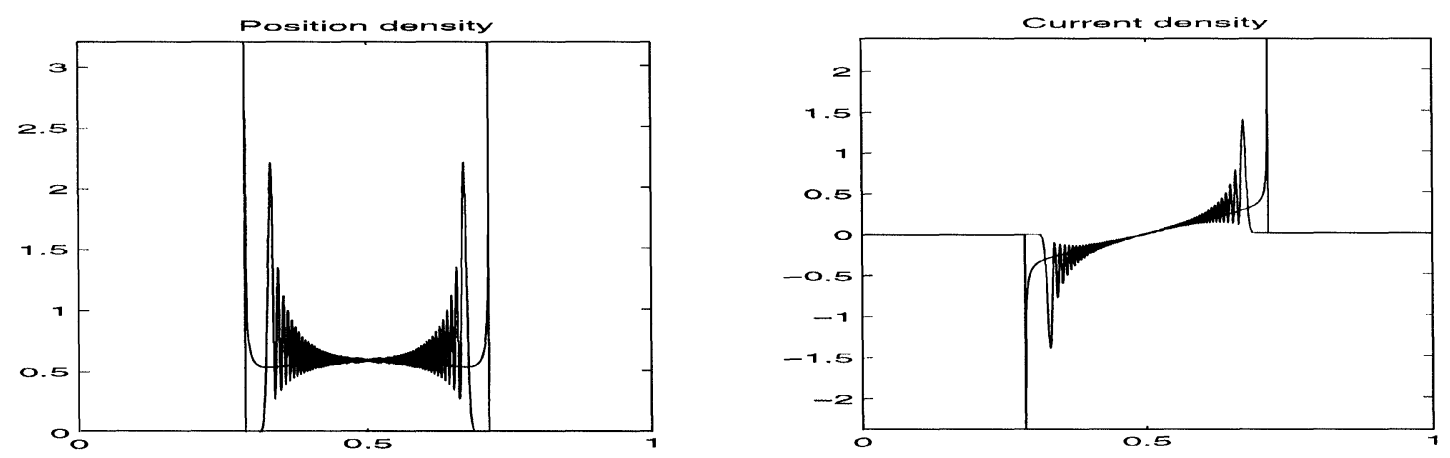

FIGURE 8 Crank-Nicolson, $\varepsilon=0.5 \cdot 10^{-3}, \Delta t=\varepsilon^{1.5}, h=\varepsilon, V=0$.
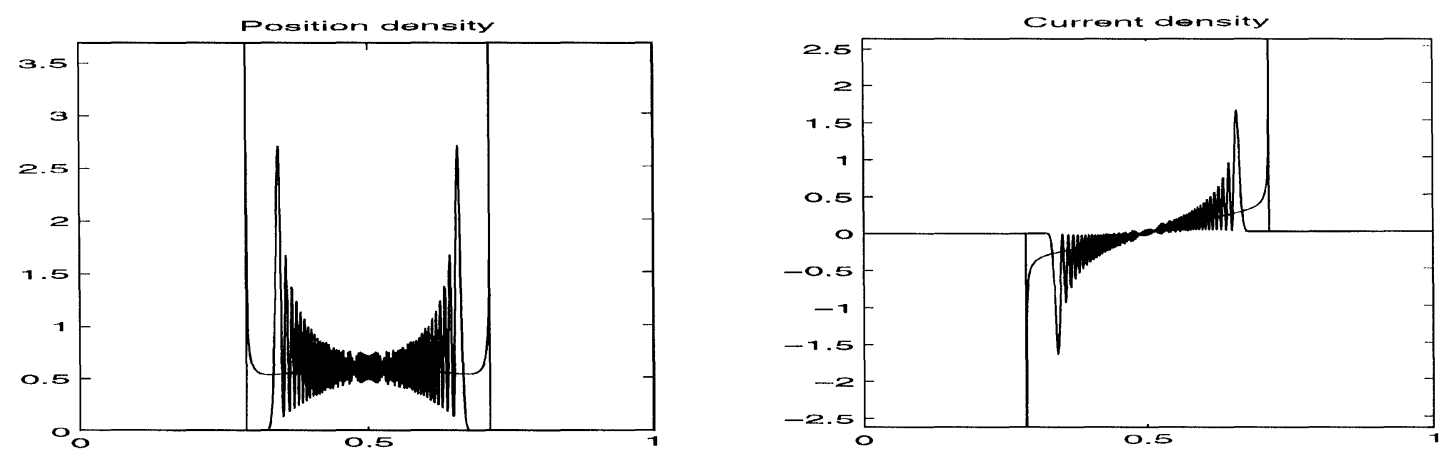

FIGURE 9 Crank-Nicolson, $\varepsilon=0.5 \cdot 10^{-3}, \Delta t=3 \varepsilon, h=\varepsilon^{1.2}, V=0$.

scheme verifies (4.5) and consequently the transport equation (2.13) (see also (5.4)) of the continuous Wigner measure. The functions $n^{\sigma}$ and $J^{\sigma}$ oscillate about $n^{0}$ and $J^{0}$ (resp.) with wave length $\varepsilon$. It is evident that when $\varepsilon$ is halved, also the wave length is halved. The amplitude of the oscillations does not grow as $\varepsilon$ becomes smaller, except the first and the last one which increase with $\varepsilon$. Thus, the pictures indicate that the sequences $\left\{n^{\sigma}\right\}$ and $\left\{J^{\sigma}\right\}$ converge weakly to $n^{0}$ and $J^{0}$. We can also say that $n^{\sigma}$ and $J^{\sigma}$ in Figures 4-6 are very good approximations of $n^{\varepsilon}$ and $J^{\varepsilon}$ for the selected $\varepsilon$ 's. 

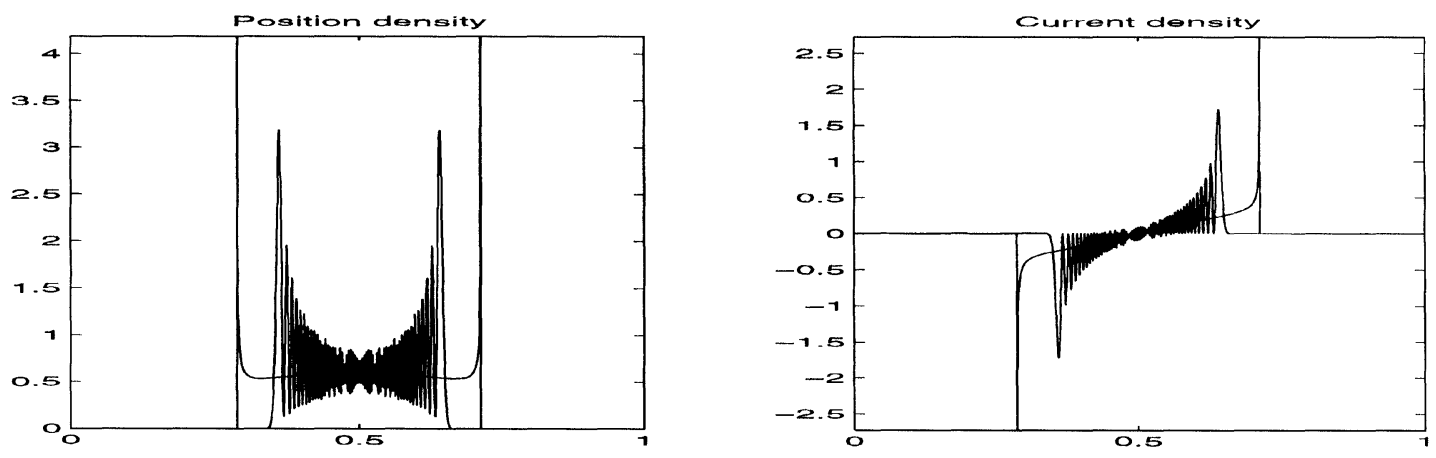

FIGURE 10 Crank-Nicolson, $\varepsilon=0.5 \cdot 10^{-3}, \Delta t=3 \varepsilon, h=\varepsilon, V=0$.
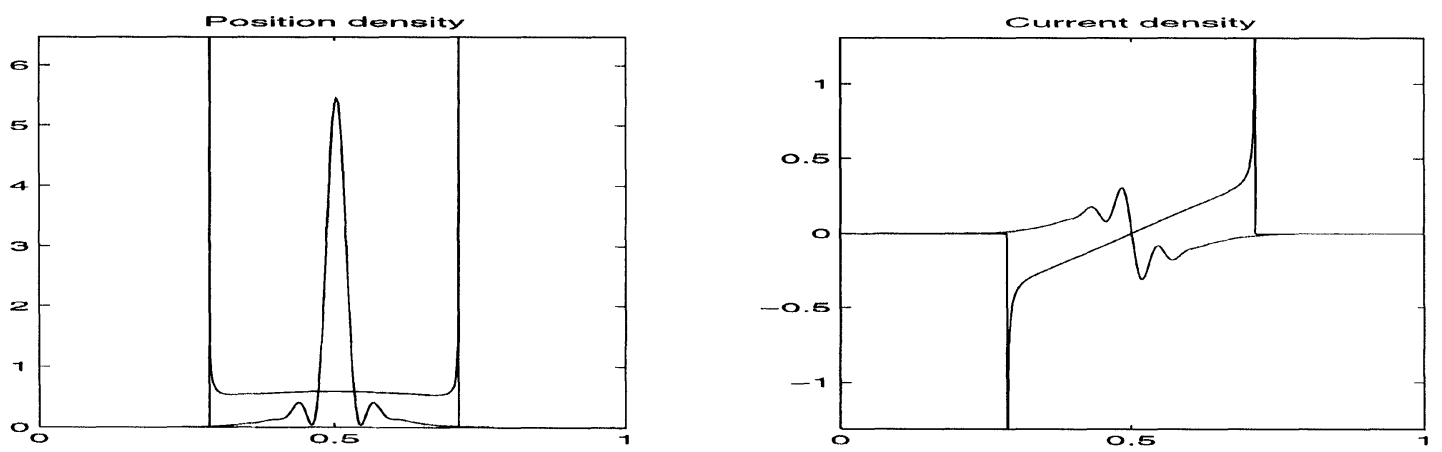

FIGURE 11 Crank-Nicolson, $\varepsilon=8 \cdot 10^{-3}, \Delta t=\varepsilon^{0.6}, h=\varepsilon^{1.2}, V=0.25$.
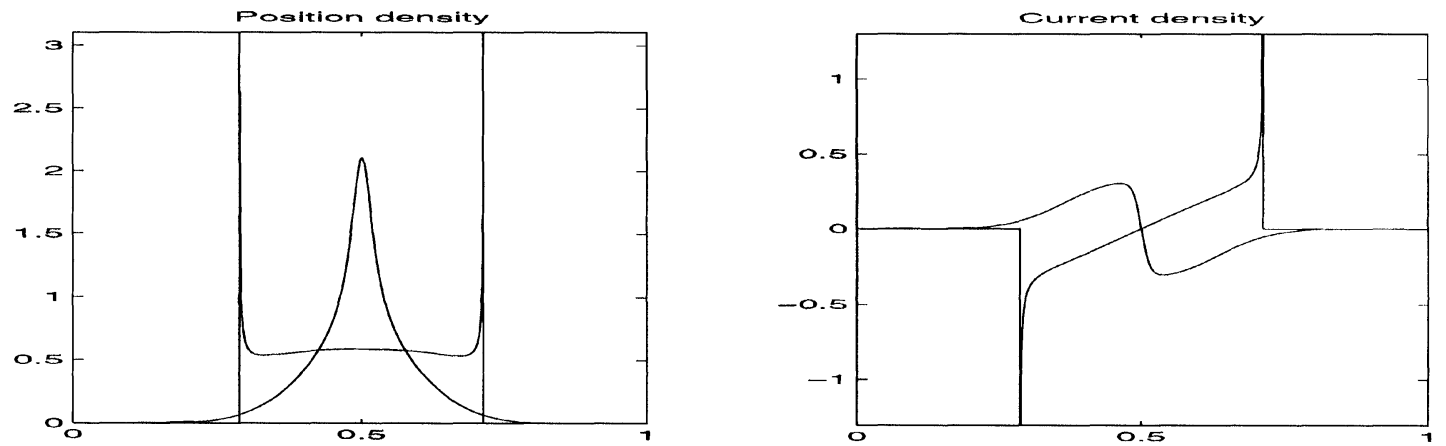

FIGURE 12 Crank-Nicolson, $\varepsilon=10^{-3}, \Delta t=\varepsilon^{0.6}, h=\varepsilon^{1.2}, V=0.25$.

When the step sizes violates (1) (i), a pathological behavior is expected, since the Wigner measure of the scheme identified in Theorem 4.1 does not coincide with the continuous one. Here we observe the effect on macroscopic quantities. We consider first the case when $\Delta t$ is still small enough $\left(\Delta t=\varepsilon^{1.5}\right)$, but the mesh size $h$ is equal to $\varepsilon$ (Case (2) (i)). Figures 7 and 8 correspond to $\varepsilon=10^{-3}$ and $\varepsilon=0.5 \cdot 10^{-3}$. The pictures indicate that $n^{\sigma}$ and $J^{\sigma}$ converge to a function with smaller support than $n^{0}$ and $J^{0}$. Moreover, comparing these plots with those obtained with $h=\varepsilon^{1.2}$, we observe that, for the same fixed $\varepsilon$, the results are qualitatively different. The support of $n^{\sigma}$ and $J^{\sigma}$ in 

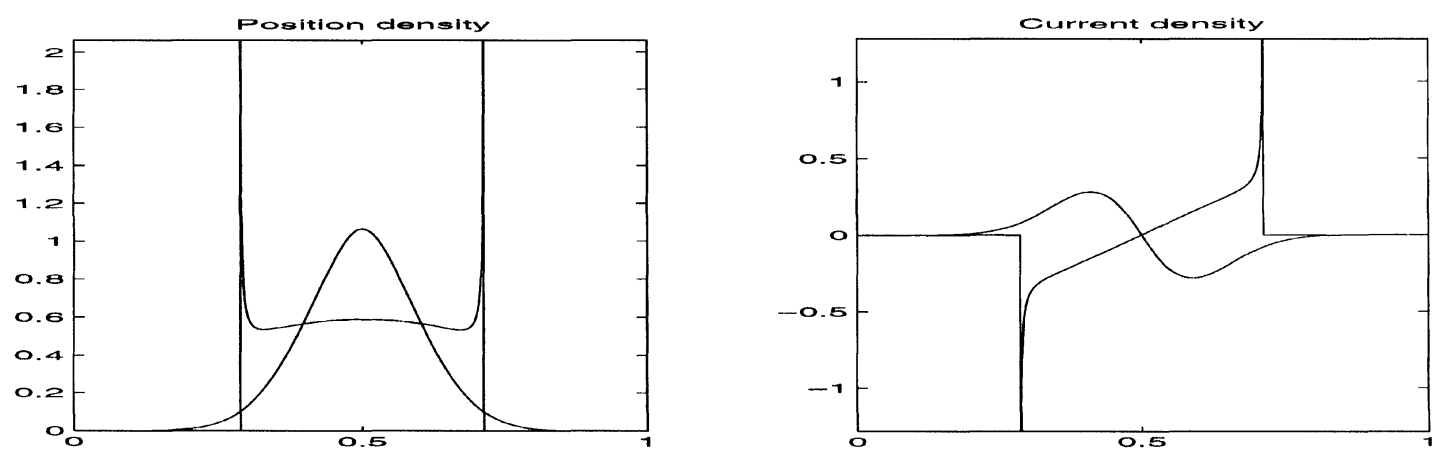

FIGURE 13 Crank-Nicolson, $\varepsilon=0.5 \cdot 10^{-4}, \Delta t=\varepsilon^{0.6}, h=\varepsilon^{1.2}, V=0.25$.
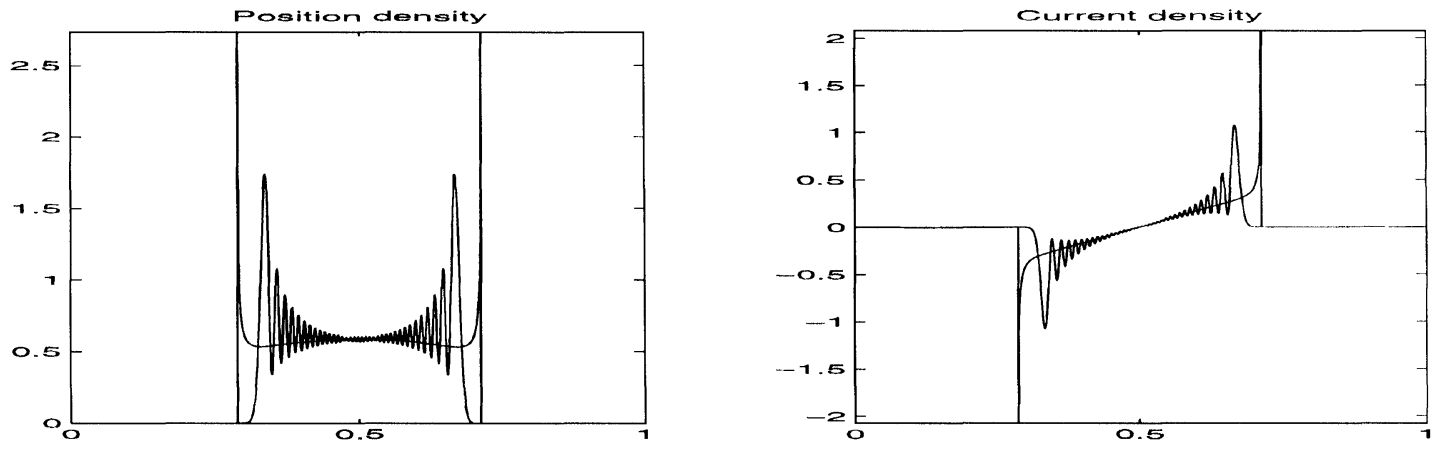

FIGURE 14 Leap-Frog, $\varepsilon=10^{-3}, \Delta t=\varepsilon^{1.25}, h=\varepsilon, V=0$.
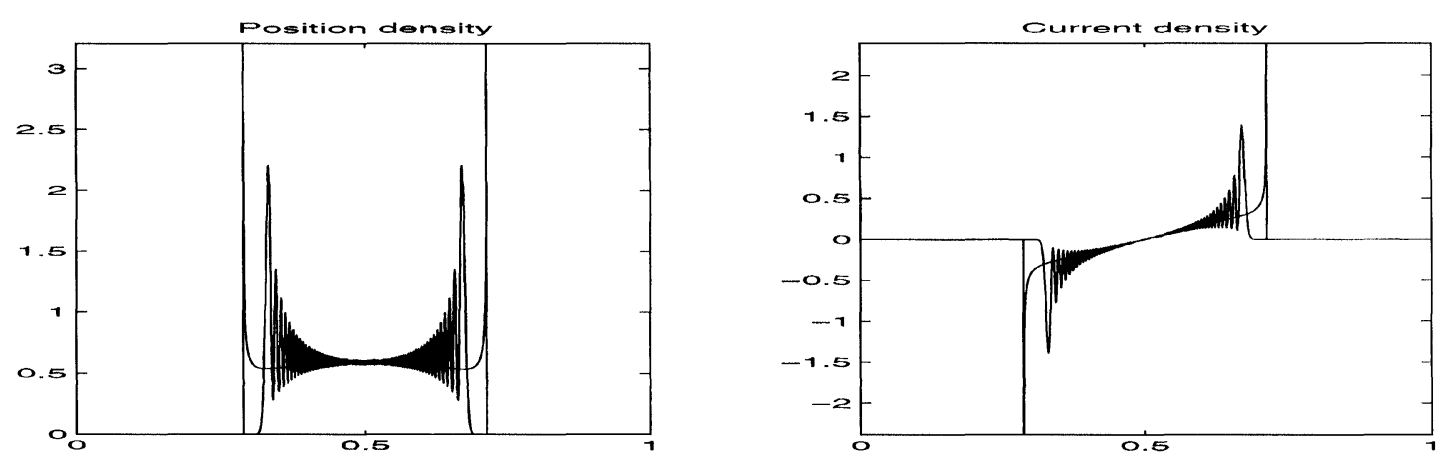

FIGURE 15 Leap-Frog, $\varepsilon=0.5 \cdot 10^{-3}, \Delta t=\varepsilon^{1.25}, h=\varepsilon, V=0$.

Figure $7,(8$, resp.) is smaller than the one of the functions in Figure 5, (6, resp.) and consequently, due to charge conservation, the oscillation amplitude is larger. $n^{\sigma}$ and $J^{\sigma}$ of Figures 7,8 are bad approximations of $n^{\varepsilon}$ and $J^{\varepsilon}$ for a fixed small $\varepsilon$. The situation is similar when $h=\varepsilon^{1.2}$ and $\Delta t=3 \varepsilon$ are selected (Case (1) (ii)). Again the solutions exhibit smaller support and higher amplitude 

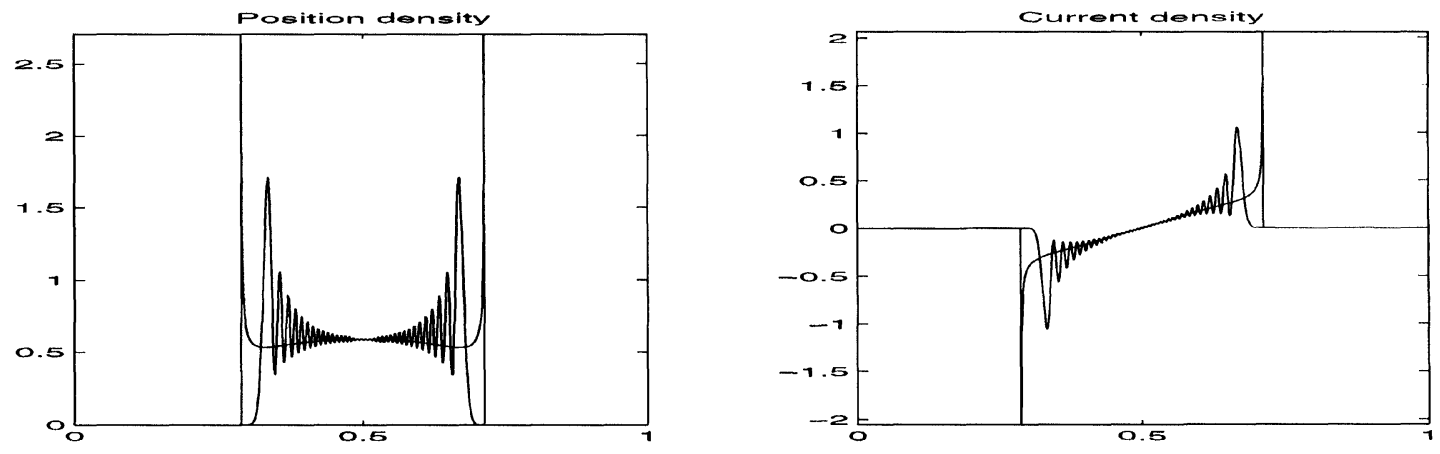

FIGURE 16 Leap-Frog, $\varepsilon=10^{-3}, \Delta t=0.4 \varepsilon, h=\varepsilon, V=0$.
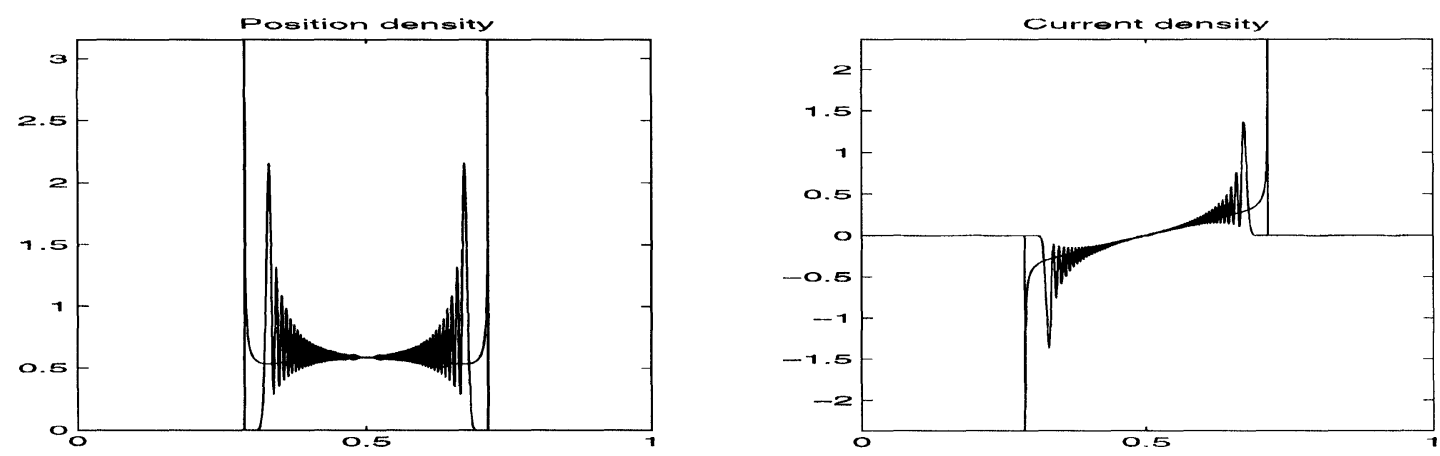

FIGURE 17 Leap-Frog, $\varepsilon=0.5 \cdot 10^{-3}, \Delta t=0.4 \varepsilon, h=\varepsilon, V=0$.

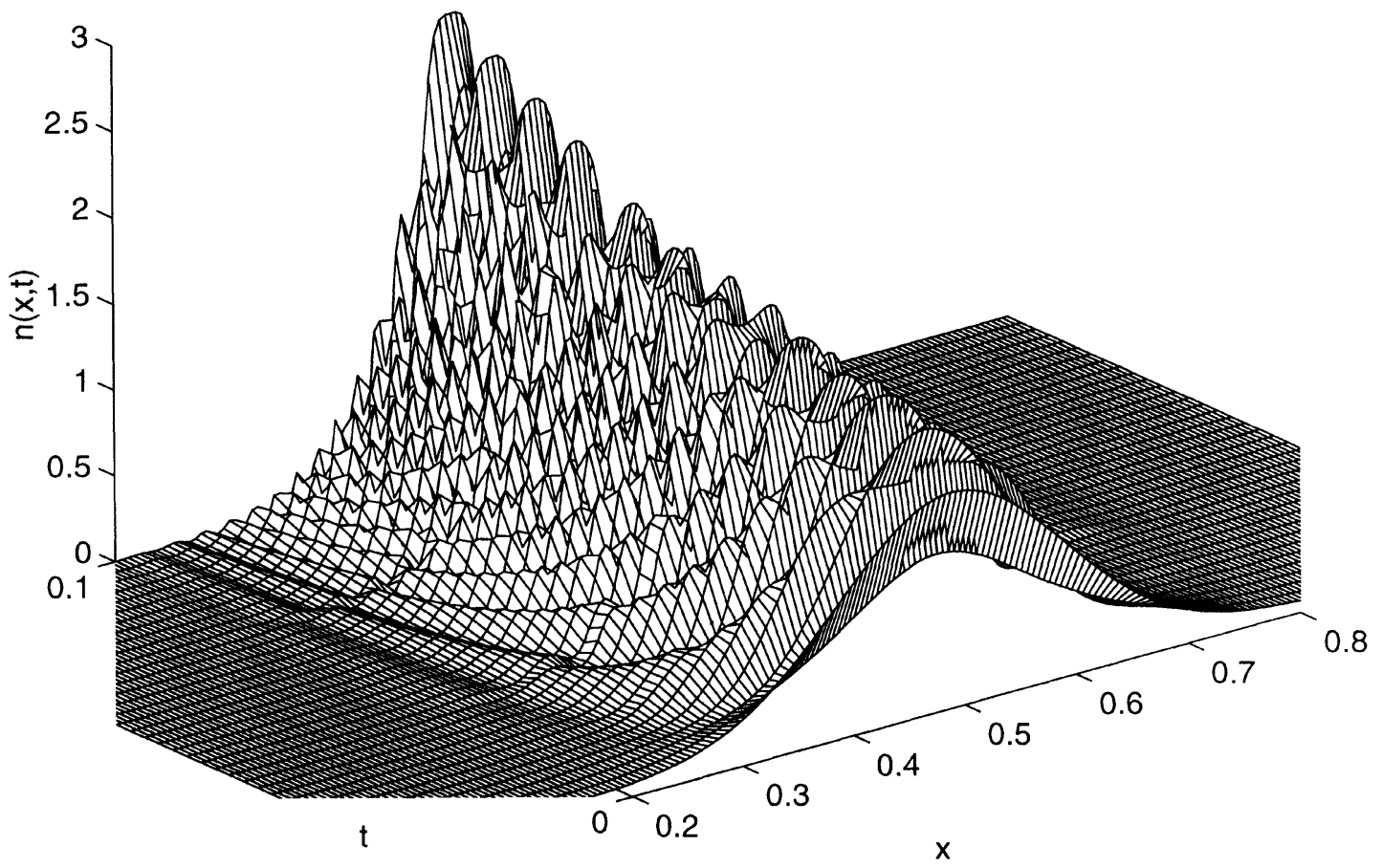

FIGURE 18 Leap-Frog, $\varepsilon=10^{-3}, \Delta t=(1 / 30) \varepsilon, h=\varepsilon, V=10,0.2<x<0.8,0 \leq t \leq 0.1$. 
oscillations (see Fig. 9, for $\varepsilon=0.5 \cdot 10^{-3}$ ). The effect is amplified when both $h$ and $\Delta t$ are too large. Figure 10 illustrates case (2) (ii) with $h=\varepsilon$, $\Delta t=3 \varepsilon$, for $\varepsilon=0.5 \cdot 10^{-3}$. In order to illustrate case (1) (iii), condition (4.6a) must be fulfilled. A constant potential $V>0$ is considered, so that $Q$ is bounded away from zero. Figures $11-13$ show the results when $V=0.25, h=\varepsilon^{1.2}, \Delta t=\varepsilon^{0.6}$, for a sequence of $\varepsilon$ 's. When $\varepsilon$ is large, (the plot for $\varepsilon=8 \cdot 10^{-3}$ is presented in Fig. 11) it is evident that $n^{\sigma}$ and $J^{\sigma}$ are far away from the correct solution, but the pictures do not suggest what the limit of the sequence is. As $\varepsilon$ becomes smaller, $n^{\sigma}$ does not exhibit oscillations and its maximum decreases with $\varepsilon$ (see Fig. 12) which refers to a computation with $\varepsilon=10^{-3}$. Figure 13 displays (for $\varepsilon=0.5 \cdot 10^{-4}$ ) $n^{\sigma}$ and $J^{\sigma}$ very close to the initial value $n_{I}$ and $J_{I}:=n_{I}(d / d x) S_{I}$, in agreement with the theoretical result $W^{0}=w_{I}$.

Leap-Frog The Leap-Frog scheme (3.5) has some interesting properties when being applied to Schrödinger type equations. It is an explicit scheme, but with a not too restrictive stability condition. For the Schrödinger equation the sharp stability bound is

$$
\frac{\Delta t}{\varepsilon}<\frac{1}{2} \frac{h^{2}}{\varepsilon^{2}+0.5 V_{\max } h^{2}},
$$

where $V_{\max }=\max _{x}|V(x)|$. Moreover, although it is not a conservative scheme it has similar properties:

- 'Charge conservation':

$$
\int \operatorname{Re}\left(u^{\sigma}\left(x, t^{n+1}\right) \overline{u^{\sigma}\left(x, t^{n}\right)}\right) d x=\text { const }
$$

- 'Energy conservation':

$$
\begin{aligned}
& \frac{\varepsilon^{2}}{2} \int \operatorname{Re}\left(\nabla_{x} u^{\sigma}\left(x, t^{n}\right) \overline{\nabla_{x} u^{\sigma}\left(x, t^{n+1}\right)}\right) d x+ \\
& \int V(x) \operatorname{Re}\left(u^{\sigma}\left(x, t^{n}\right) \overline{u^{\sigma}\left(x, t^{n+1}\right)}\right) d x=\text { const. }
\end{aligned}
$$

All the tests presented here are carried out with $\tilde{u}_{1}^{\sigma}=u_{0}^{\sigma}=u_{I}^{\varepsilon}$, so that $z_{I}^{0}=w_{I}^{0}$ (see Theorem 4.2).

Let us discuss case (1) of Theorem 4.2 first. Only a condition on $h$ is stated, since the stability condition
(5.10) implies that $(\Delta t / \varepsilon)$ goes to zero in this case. For instance, if $V=0$ is selected, then the stability condition forces $(\Delta t / \varepsilon)<0.5\left(h^{2} / \varepsilon^{2}\right)$. For $h=\varepsilon^{1.2}$ and $\Delta t=0.49 \varepsilon^{1.4}$ the stability condition is satisfied and the results for $\varepsilon=2 \cdot 10^{-3}, \varepsilon=10^{-3}, \varepsilon=$ $0.5 \cdot 10^{-3}$ are very similar to those of Figures $4-6$ (obtained with the Crank-Nicolson scheme) and are not reported. Note that in this case the stability condition allows a $\Delta t$ very close to $\Delta t=\varepsilon^{1.5}$ used in the tests with the Crank-Nicolson scheme. Of course, if $h$ is much smaller than $\varepsilon,(5.10)$ requires a much smaller $\Delta t$ than the one requested by condition (i) of Theorem 4.1 for Crank-Nicolson.

All the pathological situations are possible for $h=O(\varepsilon)$. As in the Crank-Nicolson case, when $Q_{\rho}$ appears in the transport Eq. (4.9) (case (2) (i)), $n^{\sigma}$ and $J^{\sigma}$ exhibit smaller support and higher oscillations than in the case when the exact $Q$ is present. Figures 14 and 15 show the results for $h=\varepsilon$, $\Delta t=\varepsilon^{1.25}$, with $\varepsilon=10^{-3}$ and $\varepsilon=0.5 \cdot 10^{-3}$. Comparing Figure 15 and Figure 8, which corresponds to case (2) (i) for Crank-Nicolson, no difference is observed.

In the case (2) (ii), we remark that the term $1-\omega^{2} Q_{h, \varepsilon}^{2}$ in (4.10a), with $\omega=\Delta t / \varepsilon$, is always positive when the stability condition (5.10) is satisfied.

Figures 16 and 17 show results with $\varepsilon=10^{-3}$ and $\varepsilon=0.5 \cdot 10^{-3}$ for $\Delta t=0.4 \varepsilon, h=\varepsilon$ and $V=0$ which are very similar to those of Figures 14 and 15 , since in this case $1-\omega^{2} Q_{h, \varepsilon}^{2}$ is almost 1 . Instead, when this coefficient is small, $Z^{0}$ and $W^{0}$ are significantly different and they evolve according to two different laws. The effect on the macroscopic quantities is weird. If, for example, $\varepsilon=10^{-3}, \Delta t=(1 / 30) \varepsilon, h=\varepsilon$ and $V=10$ then the maximum of $n^{\sigma}$ oscillates almost every time step. Figure 18 shows the evolution of $n^{\sigma}(x, t)$ for $0 \leq t \leq 0.1$. Although the density $n^{\varepsilon}$ is smooth in this time intervall (we recall that caustics develop at time $t=0.2$ ) the wave function $u^{\varepsilon}$ (solution of the Schrödinger equation) has oscillations in the phase. A choice of the discretization parameters that does not take care of this fact can give the wrong results. Actually, Theorems 4.1 and 4.2 apply regardless of the occurance of caustics. 


\section{Acknowledgement}

All three authors acknowledge support from the DAAD-VIGONI. The first and third authors were also supported by the DFG-project \#MA 1662/1-2. We are grateful to Andreas Unterreiter for helpful discussions.

\section{References}

[1] Chan, T. F., Lee, D. and Shen, L., Stable Explicit Schemes for Equations of the Schrödinger Type. Siam J. Numer. Anal., 23(2), April 1986, 274-281.

[2] Chan, T. F. and Shen, L., Stability Analysis of Difference Schemes for Variable Coefficient Schrödinger Type Equations. Siam J. Numer. Anal., 24(2), April 1987, 336-349.

[3] Dörfler, W. (1996). A time- and spaceadaptive algorithm for the linear time-dependent Schödinger equation. Numer. Math., 73, 419-448.

[4] Delfour, M., Fortin, M. and Payre, G. (1981). Finite-Difference Solutions of a Non-linear Schrödinger Equation. Journal of Computational Physics, 44, 277-288.

[5] Gérard, P., Mesures Semi-Classiques et Ondes de Bloch. Sém. Ecole Polythechnique (1990-1991). exposé, XVI, $1-19$.

[6] Gérard, P. (1991). Microlocal Defect Measures. Comm. PDE., 16, $1761-1794$.

[7] Patrick, Gérard, Peter, A. Markowich, Norbert, J. Mauser and Poupaud, Frédéric (1997). Homogenization Limits and Wigner Transforms. Comm. Pure and Appl. Math., 50, $321-377$

[8] Hörmander, L. (1985). The Analysis of Linear Partial Differential Operators III. Springer.

[9] Jin, Shan, David Levermore, C. and David, W. McLaughlin (1994). The Behavior of Solutions of the NLS Equation in the semiclassical Limit. Singular Limits of Dispersive Waves. Plenum Press New York and London.

[10] Landau, L. D. and Lifschitz, E. M. (1985). Lehrbuch der Theoretischen Physik III-Quantenmechanik. AkademieVerlag.

[11] Lions, P. L. and Paul, T. (1993). Sur les Mesures de Wigner. Revista Mat. Iberoamericana, 9, 553-618.

[12] Markowich, P. A. and Mauser, N. J. (1993). The Classical Limit of a Self-consistent Quantum-Vlasov Equation in 3D. Math. Mod. and Meth. in Appl. Sciences, 9, 109-124.

[13] Markowich, P. A., Mauser, N. J. and Poupaud, F. (1994). A Wigner function approach to semiclassical limits: electrons in a periodic potential. J. Math. Phys., 35, 1066-1094.

[14] Markowich, P. A. and Poupaud, F. (1997). The Pseudo-Differential Approach to Finite Differences Revisited. submitted.

[15] Markowich, P. A., Pietra, P. and Pohl, C. (1999). Numerical Approximation of Quadratic Observables of Schrödinger-type Equations in the Semi-classical Limit. Numer. Math., 81, 595-630.

[16] Pathria, D. and Morris, J. L. (1990). Pseudo-spectral Solution of Nonlinear Schrödinger Equations. Journal of Computational Physics, 87, 108-125.

[17] Strikwerda, John C. (1989). Finite Difference Schemes and Partial Differential Equations. Wadsworth \& Brooks/Cole Advanced Books \& Software. Pacific Grove, California.
[18] Sanz-Serna, J. M. and Manoranjan, V. S. (1983). A Method for the Integration in Time of Certain Partial Differential Equations. Journal of Computational Physics, 52, $273-289$.

[19] Tartar, L. (1990). H-measures: a new approach for studying homogenization, oscillations and concentration effects in partial differential equations. Proc. Roy. Soc. Edinburgh Sect. A, 115, 193-230.

[20] Taha, T. R. and Ablowitz, M. J. (1984). Analytical and Numerical Aspects of Certain Nonlinear Evolution Equations. II. Numerical, Nonlinear Schrödinger Equation. Journal of Computational Physics, 55, 203-230.

[21] Wu, L., Dufort-Frankel-Type Methods for Linear and Nonlinear Schrödinger Equations. Siam J. Numer. Anal., 33(4), August 1996, 1526-1533.

[22] Wigner, E. (1932). On the Quantum Correction for the Thermodynamic Equilibrium. Phys. Rev., 40.

\section{Authors' Biographies}

Peter A. Markowich received his Ph.D. in Applied Mathematics from the Technical University of Vienna (Austria) in 1980. He was Professor of Mathematics at the Technical University of Berlin (Germany) from 1989 to 1998 and is currently Professor of Mathematics at the University of Linz (Austria). His fields of interest are partial differential equations and kinetic theory.

Paola Pietra Born on 30.9.1955 in Pavia, Studies of Mathematics at the Università di Pavia: 1974-1978, Position: Research Director, Professional career: C.N.R. fellowship at the Istituto di Analisi Numerica, Pavia, 1979-1982, Researcher at the same institution, 1982-1991, Research Director at the same institution from 1991 to now, Postdoctoral position at the Department of Mathematics of the University of Chicago from September 1984 to October 1985, Visiting Associate Professor at the Mathematics Department of Purdue University, West Lafayette, Indiana, from August 1990 to May 1991.

Carsten Pohl Born on the 25.5.1968 in Berlin. October 1987-April 1994: Studies of mathematics at the TU Berlin, masters exam with distinction, May 1994-May 1998: Ph.D. student at the TU Berlin, since May 1998 at the Johannes Kepler University Linz, June 1998: Ph.D. exam with distinction. 

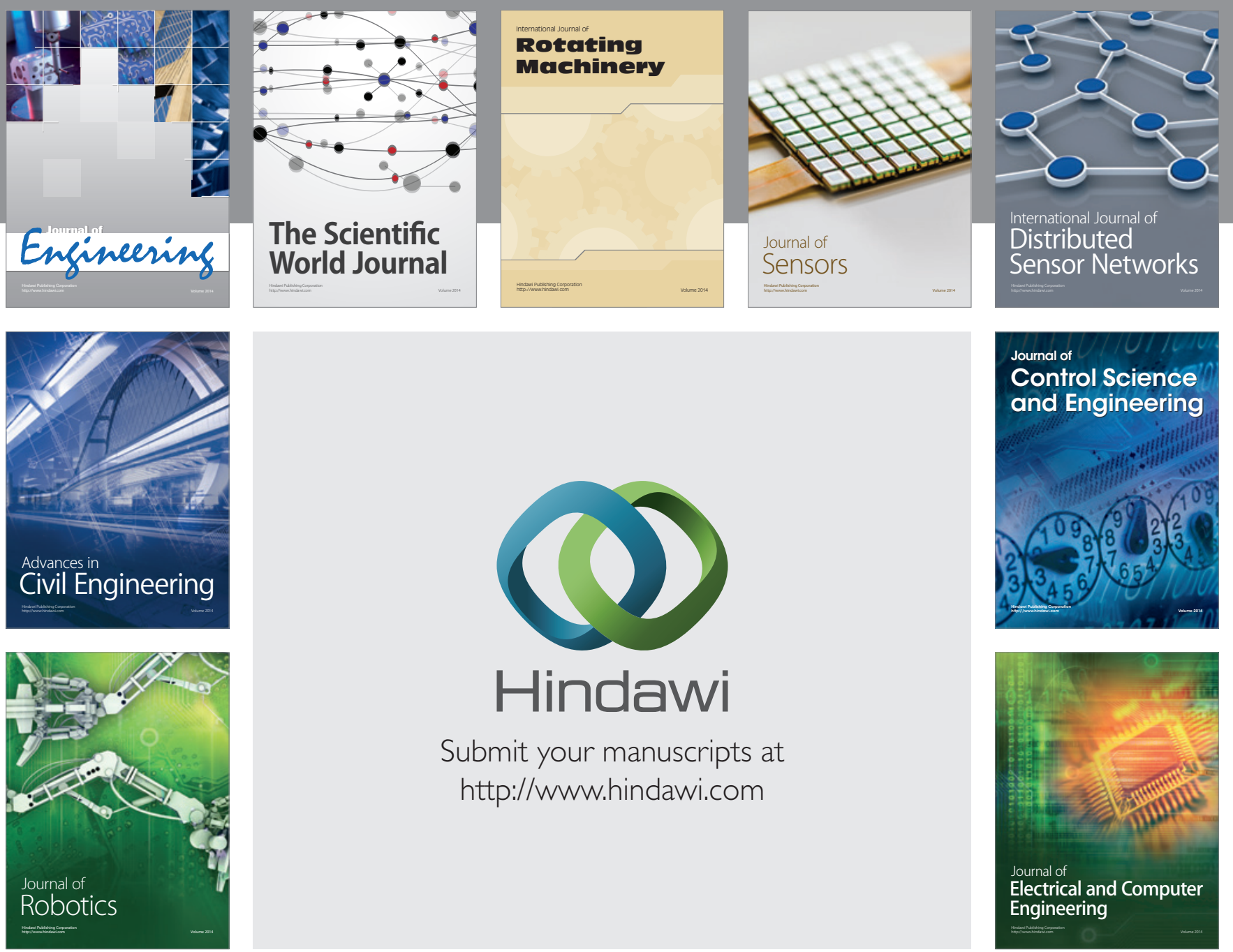

Submit your manuscripts at

http://www.hindawi.com
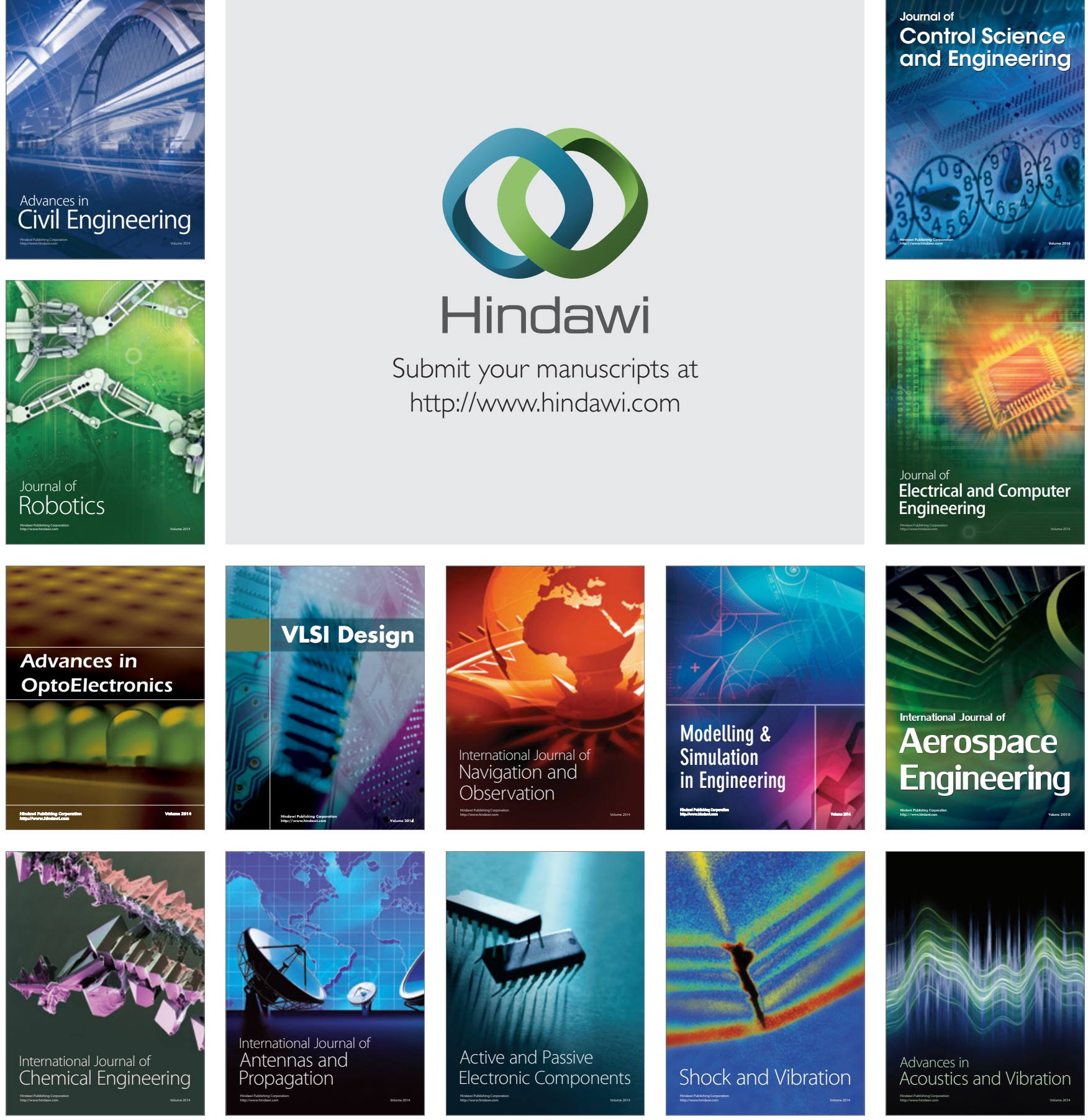